\title{
On Topological Issues of Indeterminism
}

\author{
Tomasz Placek $\cdot$ Nuel Belnap $\cdot$ Kohei Kishida
}

Received: 3 February 2013/Accepted: 3 February 2013/Published online: 14 May 2013

(C) The Author(s) 2013. This article is published with open access at Springerlink.com

\begin{abstract}
Indeterminism, understood as a notion that an event may be continued in a few alternative ways, invokes the question what a region of chanciness looks like. We concern ourselves with its topological and spatiotemporal aspects, abstracting from the nature or mechanism of chancy processes. We first argue that the question arises in Montague-Lewis-Earman conceptualization of indeterminism as well as in the branching tradition of Prior, Thomason and Belnap. As the resources of the former school are not rich enough to study topological issues, we investigate the question in the framework of branching space-times of Belnap (Synthese $92: 385-434,1992)$. We introduce a topology on a branching model as well as a topology on a history in a branching model. We define light-cones and assume four conditions that guarantee the light-cones so defined behave like light-cones of physical space-times. From among various topological separation properties that are relevant to our question, we investigate the Hausdorff property. We prove that each history in a branching model satisfies the Hausdorff property. As for the satisfaction of the Hausdorff property in the entire branching model, we prove that it is related to the phenomenon of passive indeterminism, which we describe in detail.
\end{abstract}

T. Placek $(\bowtie)$

Jagiellonian University, Kraków, Poland

e-mail: tomasz.placek@uj.edu.pl

\section{N. Belnap}

University of Pittsburgh, Pittsburgh, USA

e-mail: belnap@pitt.edu

\section{K. Kishida}

University of Amsterdam, Amsterdam, The Netherlands

e-mail: kishidakohei@gmail.com 


\section{Problem}

Uncontroversially, our world has spatial and temporal aspects, or (as the physics has it) spatiotemporal aspects. Accordingly, an adequate analysis of (in)determinism of our world must explain how (in)determinism plays out in space and time (or in a spacetime). Although the intuitive core of the notion of (in)determinism, i.e., "the past does (not) determine uniquely the future" is hardly contentious, there are a few proposals of how to rigorously explain this intuition. Prominent are two approaches nowadays, that develop, respectively, a Laplacean, physics-inspired, concept and a notion based on modal intuitions voiced perhaps first in Aristotle's De Interpretatione. Despite deep philosophical differences (which we will review shortly), the two approaches face a similar challenge: to explain what a region of indeterminism looks like. Here is how the question arises. Without making any claim to historical accuracy, it is good to think of both schools as introducing a concept of a global course of events, calling it a possible world, possible history, or possible scenario. To analyze (in)determinism, they next postulate some global directionality, which needs to be neither temporal, nor linear. As a result, the Laplacean school has a concept of initial segments of possible worlds, whereas the Aristotelian invokes a distinction between an event's past, its future of possibilities, and its wings, that is, the set of events space-like related to it. To address the question of (in)determinism, the Laplacean then looks for isomorphic initial segments of a pair of possible worlds, the later segments of which are not isomorphic. The Aristotelian school asks if there is some event whose future of possibilities contains more than one alternative possibility. Accordingly, both the schools legitimize a notion of passing from the region of determinateness (isomorphic initial segments, or an event's past including perhaps its wings) to the region of indeterminacy (one of non-isomorphic later segments, or one of alternative possible futures of an event). We may thus consider a path, say a photon's trajectory, passing from the region of determinateness to the region of indeterminacy. Since a spacetime is typically assumed to be continuous, the following question arises: What does the path look like at the border of determinateness and indeterminacy? For instance, is there a last element in the the determinateness region, or a first element in the indeterminacy region?

The proper tools to handle questions like this belong to topology, and in particular, to the theory of the so-called separation properties. Our paper focuses almost entirely on one of these properties, called the Hausdorff property, to be explained in Sect. 3.1. This property comes to the fore in debates over topological aspects of indeterminism: Supposing that indeterminism plays out in spacetime, philosophers and physicists typically ask if the Hausdorff property is satisfied. ${ }^{1}$ Our focus on the Hausdorff property has a practical dimension as well: An investigation of other separation properties would extend the size of this essay beyond any tolerable limit. Other topological features of indeterminism nevertheless require study as well. In fact, we hope this essay will serve as a call to further investigate the

\footnotetext{
${ }^{1}$ Recently the philosopher Earman (2008) discussed the Hausdorff property in relation to various ways of conceptualizing indeterminism. On the role of Hausdorff property in physics we have also been taught by the physicist A. Staruszkiewicz, whom we gratefully acknowledge.
} 
issue of what the region of passage from determinateness to indeterminacy looks like. The phrase "looks like" is to indicate that we are not concerned with the physics of indeterministic processes, that is, with the question of how indeterminism occurs. We are concerned solely with a combination of spacetime and modality, and the topological issues this combination raises.

To handle the topological questions, we need, however, a rigorous theory combining indeterminism and spacetime. With the exception of Montague's (1962) model-theoretic analysis of point mass mechanics and Newtonian gravitation, there is not enough mathematical rigor in the current developments of Laplacean (in)determinism to permit a fruitful study of topological issues. The required rigor is possessed by Belnap's (1992) axiomatic theory of branching space-times (BST), which combines (in)determinism with (rudimentary) relativistic spacetimes. Moreover, the BST axioms are frugal, and as far as topological questions are concerned, BST and the Laplacean approach have some affinity (as we show in Sect. 2). Thus, although we will carry out our investigations in the BST framework, our findings have bearing for the Laplacean school as well.

As we said, we will investigate one separation property: the Hausdorff property. In doing this, we will pursue two quite separate goals. Chiefly we address the following question: Under what conditions does a BST model of indeterminism (that is, a model of BST comprising many branching histories) satisfy the Hausdorff property? Our secondary aim is to address the Earman (2008) argument that "literal branching of a relativistic spacetime" (p. 193) leads to a failure of the Hausdorff property. ${ }^{2}$ The failure of the Hausdorff property has various negative consequences for physics, which Earman lists. So, in order to avoid these consequences, each individual spacetime in BST needs to satisfy the Hausdorff property. We thus investigate whether or not this is so. Intuitively speaking, it is unbelievable that adding indeterminism should destroy topological properties of a single spacetime (or history in BST parlance). In our earlier publication (Placek and Belnap 2012) we stated some theorems (mostly without proof) that supported the unbelievability intuition. Here we lay down our reasoning in full detail, showing that a single history satisfies the Hausdorff property, in spite of our BST-style explanation of indeterminism in terms of branching.

The essay is organized as follows. Section 2 contrasts the two schools of thinking about (in)determinism, Laplacean and Aristotelian. Section 3 introduces topology for BST and shows that each BST history satisfies the Hausdorff property. An important part of this task is the introduction of light-cones into the abstract framework of BST. Then Sect. 4 proves that each single BST history (aka spacetime) satisfies the Hausdorff property. Section 5 presents theorems exhibiting necessary and sufficient conditions for a BST branching-histories model to satisfy

\footnotetext{
${ }^{2}$ The argument does not specify with respect to which topology the Hausdorff property fails; in particular, it does not relate to the so-called Bartha topology, put forward by Belnap (1992) as a natural topology for BST. Since one may always produce a Hausdorff topology on a set (e.g., discrete topology) we read the argument as saying that on every natural topology, a branching relativistic spacetime is nonHausdorff. We counter it by showing that such a spacetime is Hausdorff with respect to the natural topology of BST, the Bartha topology.
} 
the Hausdorff property. And Sect. 6 lays out arguments that the topologies discussed in this essay are natural topologies for BST. The essay ends with Conclusions.

\section{Two Schools of (in)Determinism}

Innocent of the concept of computers or Turing machines, Laplace (1820) had recourse to a powerful intelligence to explicate a concept of determinism, the inspiration for which came from Newtonian mechanics. The intelligence is supposed to have two kinds of data, first about an instantaneous state of a system and second about a mathematical form of all forces acting in the system. These data enable the intelligence to have the future as well as the past of the system "present before its eyes." One problem with this characterization is that it is stated in epistemic terms. A natural move of replacing the metaphorical "present before its eyes" by "to predict" or "to compute" does not change this predicament. "De-epistemologizing" of Laplacean indeterminism was achieved by Montague (1962), who defined a few versions of Laplacean indeterminism in model-theoretic terms, and applied these definitions to mass point particle mechanics and Newtonian gravitation. Montague's momentous decision was to take (in)determinism to be ascribable to theories, a move that many philosophers have followed. What the successors rarely appreciate, however, is that a theory was understood by Montague in the logical sense, that is, as fully characterized by a (formal) language and a set of axioms. Thus, to apply Montague's machinery, one needs first to formulate a given physical theory in some formal language, and then to select an adequate set of axioms, that is, a set that would deliver truths and only truths of the physical theory in question. A verdict of whether a theory is deterministic is then based on the behavior of models (more precisely, partial models) of the theory. Roughly, a theory is deterministic iff whenever the initial segments of two of its partial models agree, the later segments of these models agree as well. If we identify a theory with the class of its models, we may derivatively say that such a class of models is deterministic or not. We may further define a model to be deterministic iff it belongs to a deterministic class of models. But it does not make sense on this approach to primarily ascribe either determinism or indeterminism to a single model: In a single model there is no structure capable of representing (in)determinism. The need to present a living physical theory as a formal language with a set of axioms (a formidable task indeed) is likely responsible for there being no results (as far as we know) achieved in Montague's framework (apart from his own).

Philosophers turned instead to Lewis's (1983) account, which takes inspiration from Montague's analysis, ${ }^{3}$ but is stated in terms of divergent possible worlds rather than partial models of a theory, the logical notion of a theory being replaced by a concept of laws of nature. He calls two worlds "divergent" iff they are not duplicates but an initial segment of one world and an initial segment of the other are duplicates (p. 359). Still, on Lewis's analysis, laws of nature bear a close affinity to a theory, since they are supposed to belong to all the true deductive systems with a

\footnotetext{
${ }_{3}^{3}$ Lewis gives full credit to Montague's work in this paper.
} 
best combination of simplicity and strength. (Hence, like a theory, they are linguistic objects.) Then Lewis's explication of determinism goes as follows:

First, a system $L$ of laws of nature is Deterministic iff no two divergent worlds both conform perfectly to the laws of that system. Second, a world is Deterministic iff its laws comprise a Deterministic system. Third, Determinism is the thesis that our world is Deterministic. (Lewis, 1983, p. 360)

Finally we quote an even more succinct formulation, due to Earman (1986, p. 13). In contrast to Lewis's formulation, it requires that two worlds agree at a time rather than on initial segments.

The world $w \in W$ is Laplacean deterministic just in case for any $w^{\prime} \in W$, if $w$ and $w^{\prime}$ agree at any time, then they agree for all times.

We now want to contrast Laplacean indeterminism with a modal concept of indeterminism, the essence of which is alternative possible futures. The milestones in the development of the modal concept of determinism is Kripke's letter to Prior (dated September 3, 1958 and reported by Øhrstrøm and Hasle (1995, p. 173)), and the following book and papers: Prior (1967), Thomason (1970), and Belnap (1992). Belnap (1992) characterizes this concept as follows:

Let Our World be the set of point events that are "in suitable external relations" to us. Accommodate indeterminism by including those point events that either are now future possibilities or were future possibilities. A point event, $e$, is indeterministic if $\Pi_{e}$ [i.e., the set of possibilities open at $e$ ] has more than one member. Otherwise, it is deterministic. Note that on this account it makes perfectly good sense to locate indeterminism not metaphorically in a theory, but literally in our world. It makes sense to say that Our World was indeterministic in Boston yesterday, but might not be so in Austin tomorrow. 4

With this little collection of quotes, we can see the differences between the two schools, which we organize as below:

External versus internal. The Laplacean school describes (in)determinism from an external standpoint, in an "eternal" language in which there are no indexicals, and in particular no tenses. Accordingly, this language does not allow one to draw a distinction between the possible and the actual, and considers all possibilities to be on a par. In contrast, the other project uses a language, in which an agent (a speaker) says things from her particular perspective. The perspective has both spatial and temporal location, but also a modal aspect, as it reflects what was/is/will be possible or actual-from a given perspective.

Global versus local. The first approach ascribes (in)determinism to large structures (like theories or worlds), whereas the other puts indeterminism on point events, and derivatively, on sets of point events.

\footnotetext{
${ }^{4} \Pi_{e}$ is defined as the partition of the set $H_{(e)}$ of histories containing $e$ that is induced by the relation $\equiv_{e}$ on $H_{(e)}$, understood as "two histories are undivided at $e^{\prime}: h \equiv_{e} h^{\prime}$ iff $\exists e^{*}\left(e^{*} \in h \cap h^{\prime} \wedge e<e^{*}\right)$. For more details, see Sect. 3.
} 
Modally thin versus modally thick. Each single object used in the definition of Laplacean indeterminism, be it Montague's partial model, or Earman/Lewis possible world, has in itself no structure to represent alternative possibilities. Each such object is, we say, modally thin. One gets (in)determinism by considering a class of such structures. In contrast, a model of the (axiomatic) BST theory (called Our World) typically has many structures (called 'histories'), to represent alternative possible courses of events of Our World. A BST model is modally thick.

(In)determinism of theories or of a world? As a result of the above, the first school ascribes (in)determinism to a theory or its class of models, or its laws of nature. Only derivatively can it ascribe (in)determinism to a single possible world, by considering it a member of a relevant set of possible worlds. In contrast, BST begins with defining (in)determinism of point events, and then uses it to define (in)determinism of a set of point events, or even to draw a distinction between deterministic versus indeterministic models of BST.

Ensemble branching versus BST branching. Current Laplaceans explicate (in)determinism in terms of what Earman (2008, p. 188) calls "ensemble branching." An ensemble is a collection of models of a given theory, where each model is a spacetime (typically, a differential manifold plus some extra structure) and these models are isomorphic at a time. (Isomorphism at issue is understood here as the existence of appropriate diffeomorphism between the manifolds.) In contrast to the Laplaceans' focus on many models (=spacetimes) of a physical theory, a generic model of the theory of BST contains more than one spacetime (aka possible history). Spacetimes branch in BST, since they intersect, but, emphatically, there is no branching within a single spacetime. A single branching spacetime is analyzed for instance by Penrose (1979) or by McCabe (2005), but BST has nothing to say about this concept. For more on ensemble branching versus BST, cf. Placek and Belnap (2012).

The differences run deep. ${ }^{5}$ For our task of investigating what a region of indeterminism looks like, however, there is an affinity between the two approaches. After all, two divergent worlds of Lewis's are isomorphic over some initial segments of them, with their larger initial segments being non-isomorphic. Similarly, two branching histories overlap in some initial region, and then branch off. We might be able to say more were we given the details of an isomorphism on initial segments of possible worlds. This matter, however, has not, as far as we know, been investigated in the Laplacean school. The call for providing some details about the mentioned isomorphism will be further reinforced by our results of Sect. 6.3 that show how natural a construction of a branching model (though not necessarily a BST model) out of a set of divergent worlds is.

The deep differences between the two ways of thinking about indeterminism are responsible, we believe, for confusedly taking a BST spacetime (history) to be nonHausdorff. An attempt to clarify this confusion was made by Placek and Belnap (2012); we repeat here the main points of their paper. BST branching is neither an ensemble branching nor a branching within a single spacetime (aka individual

\footnotetext{
5 For more about the controversy over modal aspects of indeterminism, see Müller (2009) or Placek and Belnap (2012).
} 
branching). BST branching relies on a modally thick notion of a theory's model. In such a model there are structures (histories or spacetimes) representing alternative courses of events. Each history/spacetime is modally thin. It does not branch in itself, that is, in BST we do not have individual branching. Placek and Belnap (2012) conjectured that in a natural topology for BST, each history/spacetime is Hausdorff (modulo some physically motivated assumptions); this claim we prove in Sect. 4. This result answers an objection of non-Hausdorfness of BST spacetimes. ${ }^{6}$

A separate issue concerns topological properties of a collection of possible worlds or histories or spacetimes. Note that such a collection is typically needed to represent indeterminism. In BST a collection of the sort in question is a multihistory model of the BST theory (with every two histories overlapping). The rigor of BST permits addressing topological issues related to the whole BST model. Regarding the Hausdorff property, Placek and Belnap (2012) claimed, and we prove here, that a multi-history BST model is non-Hausdorff in its natural topology (modulo some intuitive assumptions). We emphasize that the same topological issues, including the satisfaction of the Hausdorff property, arise in the Laplacean tradition. Is an ensemble of spacetimes, as a whole, Hausdorff? More generally, what topological properties does such an ensemble have? To handle these questions in the Laplacean tradition requires a clarification about details of constructing an ensamble, in particular, details concerning isomorphism between segments of spacetimes, as well as a choice of topology. But, if it turns out that ensembles of spacetimes are non-Hausdorff, this result in itself will not speak against the Laplacean (ensemble-style) representation of (in)determinism of spacetime theories. In a similar vein, non-Hausdorffness of a whole multi-history BST model does not speak against a branching-style representation of (in)determinism of spacetime theories.

We thus suspect that there is a different worry lurking behind topological objections to branching, namely, Is branching capable of representing indeterminism of theories of physics? This problem requires a separate investigation that lies definitely outside the scope of this study. We nevertheless give here a hint as to why we are optimistic about application of branching to physics. As Müller (2009) argues, although the current orthodoxy in philosophical approaches to determinism is Laplacean, defining (in)determinism of a theory in terms of an ensemble of the theory's models, the assessment of a theory's (in)determinism is not done in terms of models (these are typically too hard to construct). A diagnosis concerning (in)determinism is based on the behavior of solutions to a theory's equations of evolution. Does a theory admit a unique equation of evolution, given the initial data? Or perhaps it permits non-unique solutions (for the same initial data) in the sense that two solutions bifurcate, after being identical for some period of time? Such non-unique solutions are naturally viewed as branching histories. Needless to say, to turn this observation into an argument that branching is capable of representing (in)determinism of theories, we need a workable branching-style

\footnotetext{
${ }^{6}$ Earman (2008) does not necessarily lever this objection against BST. He says, "Since I have been unable to get a fix on what Belnap branching involves, all I can say for the present is this: insofar as Belnap branching eschews individual branching, then for present purposes I have no quarrel with it ..." (p. 192), and, yes, we eschew individual branching.
} 
definition of (in)determinism of a theory. The task of constructing such a definition we leave, however, for a future project.

We proceed now to investigate topological questions of indeterminism in the framework of branching space-times.

\section{Topology of BST}

"BST" stands for the theory of branching space-times as introduced in Belnap (1992) and developed by several hands, as indicated in the References.

A model of BST is defined as a dense partial order $O W=\langle W, \leq\rangle$ without maxima that satisfies two simple conditions, conditions that are more transparent if we use the following definition: A history in $O W$ is a maximal (upward) directed set; we use $h$ for histories. $h_{1} \perp_{e} h_{2}$ iff $e$ is a "choice point" for $h_{1}$ and $h_{2}$, that is, iff $e$ is a maximum of $h_{1} \cap h_{2}$. (1) Every lower bounded chain has an infimum, and every upper bounded chain has a supremum in every history that contains it; and (2) (prior choice postulate) where $h_{1}, h_{2}$ are histories in $O W$, let $E$ be a chain in $h_{1} \backslash h_{2}$. Then $\exists e(e<E$ and $\left.h_{1} \perp_{e} h_{2}\right){ }^{7}$ Later we will indicate some illuminating conditions that can be added to BST in order to make full contact with certain topological questions.

In addition to "history" and "choice point," the following defined terms prove invaluable in articulating the properties of BST.

Definition 1 (Histories) Hist is the set of all histories in $O W . H_{(e)}=\{h \in$ Hist $\mid e \in$ $h\}$ is the set of histories containing $e$.

$h \equiv_{e} h^{\prime}$ (read as "histories $h$ and $h^{\prime}$ are undivided at $e^{\prime)}$ iff $\exists e^{\prime}\left(e<e^{\prime} \wedge e^{\prime} \in\right.$ $\left.h \cap h^{\prime}\right)$. (Note that $h \equiv_{e} h^{\prime}$ is provably an equivalence relation on $H_{(e)}$.)

$h \perp_{e} h^{\prime}$ (read as " $h$ and $h^{\prime}$ divide (or split) at $e^{\prime \prime}$ ) iff $e$ is a maximum in $h \cap h^{\prime}$.

Two events $e_{1}, e_{2} \in W$ are space-like related, $e_{1} \operatorname{SLR} e_{2}$, iff they are incomparable and there is a history to which the two belong.

\subsection{BST: The Diamond Topology}

In this section we will introduce a topology for BST in order to investigate in the next sections whether the Hausdorff property is satisfied in BST histories and in BST models. Let us first recall the Hausdorff property:

Definition 2 Suppose that $\mathfrak{I}$ is a topology on set $X$. Then $\mathfrak{I}$ has the Hausdorff property iff for any two distinct $e, e^{\prime} \in X$ there are disjoint sets $U, V \in \mathfrak{I}$ such that $e \in U$ and $e^{\prime} \in V$.

We introduce now, following Bartha, what we claim to be a thoroughly natural topology for BST. $^{8}$ (See Sect. 6 for arguments for the naturalness.)

\footnotetext{
7 In considering probabilities in BST, Weiner and Belnap (2006) recorded a need (discovered by Weiner) for a further natural postulate. It plays no role, however, in our current investigation.

${ }^{8}$ Cf. Belnap (2003a), note 26.
} 
Definition 3 (diamonds) $\quad$ Let $O W=\langle W, \leqslant\rangle$ be a BST model. We define

$$
d^{e_{1} e_{2}}:=\left\{y \in W \mid e_{1}<e_{2} \wedge e_{1} \leqslant y \leqslant e_{2}\right\},
$$

which we call "the diamond with vertices $e_{1}$ and $e_{2}$."

Further, if want to stress that vertices $e_{1}, e_{2}$ belong to a certain maximal chain $t$ in $W(t \in M C(W))$, we will write $d_{t}^{e_{1} e_{2}}\left(=d^{e_{1} e_{2}}\right)$, calling it "the diamond oriented by $t$ with vertices $e_{1}$ and $e_{2} . "$

The 'diamond' terminology reflects the fact that the above condition, if applied to $\mathbb{R}^{2}$ ordered by the Minkowskian ordering, yields diamond-like shapes. ${ }^{9}$

Definition 4 (the diamond topology on $W$ ) $Z$ is an open subset of $W, Z \in \mathfrak{I}(W)$, iff $Z=W$ or for every $e \in Z$ and for every $t \in M C(W)$ containing $e$ there is a diamond $d_{t}^{e_{1} e_{2}} \subseteq Z$ that is oriented by $t$ with $e$ strictly between the diamond's vertices $e_{1}$ and $e_{2}$.

Thus, $Z \in \mathfrak{I}(W)$ iff $Z=W$ or

$$
\forall e \in Z \quad \forall t \in M C(W)\left(e \in t \rightarrow \exists e_{1}, e_{2} \in t\left(e_{1}<e<e_{2} \wedge d_{t}^{e_{1} e_{2}} \subseteq Z\right)\right) .
$$

The condition of the above definition can be used to define the topology $\mathfrak{I}(h)$ on history $h \in$ Hist as well:

Definition 5 (the diamond topology on a single history) $Z \in \mathfrak{I}(h)$ iff $Z=h$ or

$$
\forall e \in Z \quad \forall t \in M C(h)\left(e \in t \rightarrow \exists e_{1}, e_{2} \in t\left(e_{1}<e<e_{2} \wedge d_{t}^{e_{1} e_{2}} \subseteq Z\right)\right),
$$

where $M C(h)$ denotes the set of maximal chains in $h$.

Despite apparent similarity, the two topologies are different, as evidenced by this fact: ${ }^{10}$

Fact 6 If $Z \subseteq h$ for some history $h \subseteq W$ contains a choice point for $h$ and some $h^{\prime}$, then $Z \notin \mathfrak{I}(W)$. However, $Z$ may belong to $\mathfrak{I}(h)$.

Proof Let $Z \subseteq h$ and $c \in h$ be a choice point for $h$ and $h^{\prime}$ and $c \in Z$. There is thus $t \in M C(W)$ such that $c \in t$ and $\forall e(e \in t \wedge c<e \rightarrow e \notin h)$. Accordingly, there are no $e_{1}, e_{2} \in t$ such that $e_{1}<c<e_{2}$ and $d_{t}^{e_{1} e_{2}} \subseteq h$. Hence for these $t$ and $c$, there are no $e_{1}, e_{2} \in t$ such that $e_{1}<c<e_{2}$ and $d_{t}^{e_{1} e_{2}} \subseteq Z$, which shows that $Z \notin \mathfrak{I}(W)$.

It follows that if $W$ is a multi-history model, its histories are not open in the topology $\mathfrak{I}(W)$. On the other hand, $\mathfrak{I}(W)$ is "coherent" with the family of topologies $\mathfrak{I}(h):^{11}$

\footnotetext{
9 The Minkowskian ordering $\leqslant \leqslant_{M}$ on $\mathbb{R}^{n+1}(n \geqslant 1)$ is defined as $x \leqslant \leqslant_{M} y$ iff $x_{0} \leqslant y_{0}$ and $\sum_{1 \leqslant k \leqslant n}\left(x_{k}-y_{k}\right)^{2} \leqslant\left(x_{0}-y_{0}\right)^{2}$, where $x=\left(x_{0}, x_{1}, \ldots, x_{n}\right), y=\left(y_{0}, y_{1}, \ldots, y_{n}\right)$, and the first coordinates are temporal.

${ }^{10}$ In fact, the topologies are different in a more significant manner than Fact 6 attests; namely, $\mathfrak{I}(h)$ may even be properly finer than the subspace of $\mathfrak{I}(W)$ on $h$. In other words, some $Z \in \mathfrak{I}(h)$ may not only fail to be in $\mathfrak{I}(W)$ itself, but also fail to have any $Z_{0} \in \mathfrak{I}(W)$ such that $Z_{0} \cap h=Z$. We nonetheless omit a proof of this fact since it is less relevant than Fact 6 to the purpose of this article.

11 See, e.g., Willard (1970, pp. 68f.), for coherent topologies.
} 
Theorem $7 \quad A \in \mathfrak{I}(W)$ iff $\forall h \in H i s t A \cap h \in \mathfrak{I}(h)$.

Proof To the right: Note that if $A \cap h=\varnothing$, then $A \cap h \in \mathfrak{I}(h)$. Pick thus an arbitrary $h \in$ Hist such that $A \cap h \neq \varnothing$. Pick then an arbitrary $e \in A \cap h$ and an arbitrary (i) $t \in$ $M C(h)$ such that $e \in t$. Since $M C(h) \subseteq M C(W)$ and $A \in \mathfrak{I}(W)$, there are (ii) $e_{1}, e_{2} \in t$ such that (iii) $d_{t}^{e_{1} e_{2}} \subseteq A$. Then $e_{2} \in h$ (by (i) and (ii)). Since histories are downward closed and $d_{t}^{e_{1} e_{2}} \leqslant e_{2}, d_{t}^{e_{1} e_{2}} \subseteq h$. Together with (iii) this implies $d_{t}^{e_{1} e_{2}} \subseteq A \cap h$.

To the left: Choose an arbitrary $e \in A$. We need to show that for an arbitrary $t \in M C(W)$ such that $e \in t$ there is a diamond $d_{t}^{e_{1} e_{2}} \subseteq A$. By the definition of histories, for every $t \in M C(W)$ there is some history $h$ such that $t \subset h$, so $t \in M C(h)$. Clearly, $e \in A \cap h$ and (by the premise) $A \cap h \in \mathfrak{I}(h)$. It follows (since $t \in M C(h)$ ) that there are $e_{1}, e_{2} \in t$ such that $e_{1}<e<e_{2}$ and $d_{t}^{e_{1} e_{2}} \subseteq A \cap h$, and hence $d_{t}^{e_{1} e_{2}} \subseteq A$.

A significant consequence of this theorem will be discussed extensively in Sect. 6.3 .

We need to check that indeed the families $\mathfrak{I}(W)$ and $\mathfrak{I}(h)$ of open sets, as defined above, form topologies. This means, in the case of $\mathfrak{I}(h)$, that $\varnothing \in \mathfrak{I}(h), h \in \mathfrak{I}(h)$, if $U, V \in \mathfrak{I}(h)$ then $U \cap W \in \mathfrak{I}(h)$, and the union of every family of sets from $\mathfrak{I}(h)$ belongs to $\mathfrak{I}(h)$. It is straightforward to see that the first two conditions are satisfied, whereas the facts below show that the remaining conditions are satisfied as well.

Fact 8 For any history $h$, if $U, V \in \mathfrak{I}(h)$, then $U \cap V \in \mathfrak{I}(h)$.

Proof Suppose $U, V \in \mathfrak{I}(h)$. If $U=h$ then $U \cap V=V \in \mathfrak{I}(h)$; similarly if $V=h$. So suppose $U \neq h$ and $V \neq h$. We need to prove that $\forall e \in U \cap V \forall t \in M C(h)\left(e \in t \rightarrow \exists e_{1}, e_{2} \in t\left(e_{1}<e<e_{2} \wedge d_{t}^{e_{1} e_{2}} \subseteq U \cap V\right)\right)$.

To this end pick an arbitrary $t \in M C(h)$ that passes through $e$. Since each of $U, V \neq h$ is open, there are diamonds $d_{t}^{a_{1} a_{2}} \subseteq U$ and $d_{t}^{b_{1} b_{2}} \subseteq V$, with $a_{1}<e<a_{2}$ and $b_{1}<e<b_{2}$. Put $e_{1}:=\max \left\{a_{1}, b_{1}\right\} \in t$ and $e_{2}:=\min \left\{a_{2}, b_{2}\right\} \in t$. Clearly, $e_{1}<e<e_{2}$. Since $d_{t}^{e_{1} e_{2}} \subseteq d_{t}^{a_{1} a_{2}} \subseteq U$ and $d_{t}^{e_{1} e_{2}} \subseteq d_{t}^{b_{1} b_{2}} \subseteq V, d_{t}^{e_{1} e_{2}} \subseteq U \cap V$.

Fact 9 For any history $h$, if $V_{\alpha} \in \mathfrak{I}(h)$ for every $\alpha \in I, \bigcup_{\alpha \in I} V_{\alpha} \in \mathfrak{I}(h)$.

Proof Suppose the antecedent. If $V_{\alpha}=h$ for some $\alpha \in I$ then $\bigcup_{\alpha \in I} V_{\alpha}=h \in \mathfrak{I}(h)$. So suppose $V_{\alpha} \neq h$ for all $\alpha \in I$. We need to prove that

$$
\forall e \in \bigcup_{\alpha \in I} V_{\alpha} \quad \forall t \in M C(h)\left(e \in t \rightarrow \exists e_{1}, e_{2} \in t\left(e_{1}<e<e_{2} \wedge d_{t}^{e_{1} e_{2}} \subseteq \bigcup_{\alpha \in I} V_{\alpha}\right)\right) \text {. }
$$

Pick an arbitrary $e \in \bigcup V_{\alpha}$; then for some $\beta \in I, e \in V_{\beta}$. Since $V_{\beta} \neq h$ is open, for every $t \in M C(h)$ such that $e \in t$ there is a diamond, $d_{t}^{e_{1} e_{2}} \subseteq V_{\beta}$, with $e_{1}, e_{2} \in t$ and $e_{1}<e<e_{2}$. But then obviously $d_{t}^{e_{1} e_{2}} \subseteq \bigcup V_{\alpha}$.

We thus proved that $\mathfrak{I}(h)$ is a topology on $h$. Although the fact that $\mathfrak{I}(W)$ is a topology on $W$ can be analogously shown by a minor tinkering in the proofs above, it also follows from the fact that $\mathfrak{I}(h)$ are all topologies, since Theorem 7 guarantees that Facts 8 and 9 imply their $\mathfrak{I}(W)$ versions: 
Fact 10 If $U, V \in \mathfrak{I}(W)$, then $U \cap V \in \mathfrak{I}(W)$.

Proof For each $h \in$ Hist, if $U \cap h, V \cap h \in \mathfrak{I}(h)$ then $(U \cap V) \cap h \in \mathfrak{I}(h)$.

Fact 11 If $V_{\alpha} \in \mathfrak{I}(W)$ for every $\alpha \in I, \bigcup_{\alpha \in I} V_{\alpha} \in \mathfrak{I}(W)$.

Proof For each $h \in$ Hist, if $V_{\alpha} \cap h \in \mathfrak{I}(h)$ for all $\alpha \in I$ then $\left(\bigcup_{\alpha \in I} V_{\alpha}\right) \cap h=\bigcup_{\alpha \in I}\left(V_{\alpha} \cap h\right) \in \mathfrak{I}(h)$.

In what follows, we need a set of particularly simple open subsets of $\mathfrak{I}(h)$ (which may not be open subsets of $\mathfrak{I}(W)$ ), to be thought of as borderless diamonds (Definition 23 below). To this end, we first need to introduce BST light-cones.

\subsection{Light-Cones in BST}

To get a better grip on the diamond topology, we would like to single out some particularly simple open subsets; ideally these subsets should form a base for the diamond topology on a history. Given that topology, a natural candidate for these subsets is borderless diamonds. A borderless diamond is a diamond from which the surface of the future light-cone of its bottom vertex and the surface of the backward light-cone of its top vertex have been removed. Accordingly, to define borderless diamonds, we need to introduce light-cones to BST. This task leads to parallel developments. On the one hand, we will define light-cones in BST and assume some conditions to guarantee that they behave at least somewhat like light-cones of spacetimes of physics. (With reference to Fig. 1, this is illustrated in items (i)-(v).) On the other hand, these conditions are shown to play a topological role, entailing ultimately that borderless diamonds indeed form a base for the topology $\mathfrak{I}(h)$.

As a warm-up, let us look at some particular segments of maximal chains asking whether they are nonempty and lower bounded. Consider first $t^{\geqslant e_{1}}:=\left\{x \in t \mid e_{1} \leqslant x\right\}$, where $t \in M C(h), e_{1} \in h$, and assume it is not empty. Then, since $t^{\geqslant e_{1}}$ is lower bounded by $e_{1}$, it has an infimum. Consider next $t^{\leqslant e_{1}}:=\left\{x \in t \mid x \leqslant e_{1}\right\}$, where $t \in$

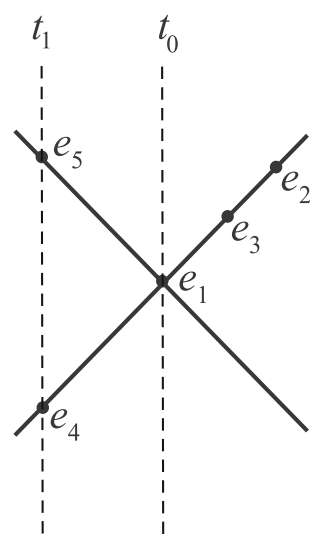

Fig. 1 Visualization of light-cones and their properties $C 1-C 4$ 
$M C(h), e_{1} \in h$ and assume it is not empty. Since clearly $t^{\leqslant e_{1}}$ is upper bounded by $e_{1}$, it has a history-relative supremum $\sup _{h}\left(t^{\leqslant e_{1}}\right)$.

Definition 12 (light-cones) Let $e_{1}, e_{2} \in h$ for some history $h \subseteq W$. We say that $e_{2}$ lies on the forward light-cone of $e_{1}$ in history $h, e_{2} \in f l c_{h}\left(e_{1}\right)$, iff $\exists t \in M C(h)\left(e_{2} \in t \wedge e_{2}=\inf \left(t^{\geqslant e_{1}}\right)\right)$.

And $e_{1}$ lies on the backward light-cone of $e_{2}$ in history $h, e_{1} \in b_{l} c_{h}\left(e_{2}\right)$, iff $\exists t \in M C(h)\left(e_{1} \in t \wedge e_{1}=\sup _{h}\left(t^{\leqslant e_{2}}\right)\right)$.

Note that by this definition $e$ lies both on the backward light-cone as well as on the forward light-cone of itself. Some further properties of the light-cones are expressed in the following Fact:

Fact 13 (about light-cones) (1) If $e_{2} \in f l c_{h}\left(e_{1}\right)$, then $e_{1} \leqslant e_{2}$; and (2) if $e_{1} \in \operatorname{blc}_{h}\left(e_{2}\right)$, then $e_{1} \leqslant e_{2}$.

Proof (1) From the antecedent, $\exists t \in M C(h)\left(e_{2}=\inf \left(t^{\geqslant e_{1}}\right)\right)$. Also, $e_{1} \leqslant t^{\geqslant e_{1}}$ follows. Since $e_{1}$ is the chain's lower bound, we obtain $e_{1} \leqslant \inf \left(t^{\geqslant e_{1}}\right)=e_{2}$. The argument for (2) is analogous.

With our definitions of lying on a light-cone, we can accommodate one of two orderings that are typically extracted from the structure of a relativistic spacetime. They are called $J$ and $I$ orderings_ c cf. Wald (1984). To put it simply, $y$ is $J$-above $x$ iff $y$ is within and including the surface of the future light-cone of $x$. Hence, an event on the surface of the future light-cone of $x$ is above $x$. In contrast, $y$ is $I$-above $x$ iff $y$ is within but excluding the surface of the future light-cone of $x$. Accordingly, an event on the surface of the future light-cone of $x$ is not above $x$. Since by Fact 13 (1-2), (the surface of) the future light-cone of $e$ is above $e$, and (the surface of) the backward light-cone of $e$ is below $e$, with our definitions of blc and $f l c$, the BST ordering is $J$, not $I$ ordering.

It is still instructive to see why Minkowski space-time with I-ordering does not yield a BST model. Consider $\mathbb{R}^{n}$ with Minkowskian $I$-ordering $\leqslant_{M}^{I}$ resulting from its irreflexive companion relation $<_{M}^{I}$, the latter being defined as: ${ }^{12}$

$$
x<_{M}^{I} y \text { iff } x_{0}<y_{0} \wedge\left(x_{0}-y_{0}\right)^{2}>\sum_{i=1}^{n-1}\left(x_{i}-y_{i}\right)^{2} .
$$

Pick a vertical chain $C=\left\{\left(z_{0}, 0,0,0\right) \mid 0<z_{0}\right\}$ approaching $O=(0,0,0,0)$ from above. $O$ lower bounds $C$ with respect to $\leqslant_{M}^{I}$, as does any $x$ such that $x_{0} \leqslant 0$ and $\sum_{i=1}^{n} x_{i}^{2} \leqslant x_{0}^{2}$. But $O$ is not an infimum of $C$ because it is not (weakly) above lower bounds of $C$ lying on the backward light-cone of $O$ (i.e., such $x$ 's that satisfy $x_{0}<0$ and $\sum_{i=1}^{n} x_{i}^{2}=x_{0}^{2}$ ). Furthermore, by applying density of $\mathbb{R}^{n}$, it is easy to note that no other element of $\mathbb{R}^{n}$ is an infimum of $C$. Thus, $\left\langle\mathbb{R}^{n}, \leqslant_{M}^{I}\right\rangle$ is not a BST model.

It turns out that for interesting topological results in branching spacetimes, one must add to the postulates of BST a group of four properties, $C 1, C 2, C 3$, and $C 4$, to be satisfied by each history of a BST model. With the help of (i)-(v) below, look at

$\overline{12}$ That is, $x \leqslant_{M}^{I} y$ iff $x<_{M}^{I} y \vee x=y$. 
Fig. 1's annotated picture of light-cones in $\mathbb{R}^{2}$. One can hardly help "seeing" that the natural properties $C 1-C 4$ of light-cones in Minkowski spacetimes (defined as Conditions 13, 15, 16, and 17 below) can be expressed in the order-theoretic vocabulary of BST.

(i) Visual verification of the propriety of Definition 12 of BST light-cones:

$$
\begin{aligned}
& \exists t_{1} \in M C\left(\mathbb{R}^{2}\right)\left(e_{5}=\inf \left(t_{1}^{\geqslant e_{1}}\right) \text { iff } e_{5} \in f l c_{h}\left(e_{1}\right)\right) \\
& \exists t_{1} \in M C\left(\mathbb{R}^{2}\right)\left(e_{4}=\sup _{\mathbb{R}^{2}}\left(t_{1}^{\leqslant e_{1}}\right) \text { iff } e_{4} \in \operatorname{blc}_{h}\left(e_{1}\right)\right)
\end{aligned}
$$

(ii) Reciprocity of $f l c$ and blc (implied by $C 1$ ):

$$
e_{2} \in f l c_{h}\left(e_{1}\right) \text { iff } e_{1} \in \operatorname{blc}_{h}\left(e_{2}\right)
$$

(iii) Betweenness $(C 2)$ :

$$
\text { if } e_{2} \in f l c_{h}\left(e_{1}\right) \wedge e_{1}<e_{3}<e_{2} \text { then } e_{3} \in f l c_{h}\left(e_{1}\right) \cap b l c_{h}\left(e_{2}\right)
$$

(iv) Interior of light-cones (C3):

$$
\exists t_{0} \in M C\left(\mathbb{R}^{2}\right)\left(e_{1} \in t_{0} \wedge t_{0} \cap\left(f l c_{h}\left(e_{1}\right) \cup b l c_{h}\left(e_{1}\right)\right)=\varnothing\right)
$$

(v) Limits of light-cones (C4):

A limit of a chain lying on a light-cone lies on the light-cone as well (if it exists).

Coming on top of Definition 12, the properties $C 1-C 4$ form a set that gives us the minimum wherewithal for making a useful connection between the order-based theory BST on the one hand, and standard Minkowski theory on the other. We shall be introducing them one by one, and sometimes considering them separately in order to tease out their several consequences; one should bear in mind, however, that they form a package. We motivate them individually by indicating what "goes wrong" in the absence of each.

Observe first the following odd fact:

Fact 14 In general, it is not true that $e_{2} \in f l c_{h}\left(e_{1}\right)$ iff $e_{1} \in b l c_{h}\left(e_{2}\right)$.

Proof See Fig. 2.

The gerrymandered history of Fig. 2 suggests that there is not enough space in it: There are no events SLR to $e_{1}$ that are immediately to the right of $e_{1}$. The two conditions below are to ensure that there is always enough space in every history:

Condition 15 ( $C 1$ : enough space) Let $h$ be a history. Then:

$$
\begin{aligned}
& \forall e_{1}, e_{2} \in h\left(\text { if } e_{2} \in f l c_{h}\left(e_{1}\right) \text {, then } \exists t \in M C(h)\left(e_{1} \in t \wedge \sup _{h}\left(t^{\leqslant e_{2}}\right)=e_{1}\right)\right), \\
& \forall e_{1}, e_{2} \in h\left(\text { if } e_{1} \in b l c_{h}\left(e_{2}\right) \text {, then } \exists t \in M C(h)\left(e_{2} \in t \wedge \inf \left(t^{\geqslant e_{1}}\right)=e_{2}\right)\right) .
\end{aligned}
$$

To see that the infima and suprema occurring in this condition exist, note that since $e_{2} \in f l c_{h}\left(e_{1}\right)$ implies $e_{1} \leqslant e_{2}, t^{\leqslant e_{2}}$ is nonempty; it is also upper bounded by $e_{2}$, so it has history-relative suprema. By a similar argument, $t^{\geqslant e_{1}}$ has an infimum. 


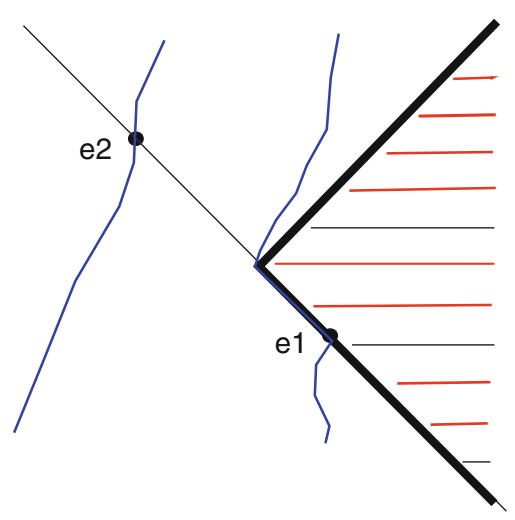

Fig. 2 This pathological one-history BST model is a part of the plane to the left of the barred area (the heavy line is included in the history). $e_{2}$ lies on the forward light-cone of $e_{1}$, but $e_{1}$ does not lie on the backward light-cone of $e_{2}$

Lemma 16 (Reciprocity) Let a history $h$ satisfy condition $C 1$ of enough space. Then $e_{2} \in f l c_{h}\left(e_{1}\right)$ iff $e_{1} \in \operatorname{blc}_{h}\left(e_{2}\right)$.

Proof Assume the LHS of the equivalence. This implies (by the first part of $C 1$ ) that there is $t \in M C(h)$ such that $e_{1} \in t \wedge \sup _{h}\left(t^{\leqslant e_{2}}\right)=e_{1}$. By Definition 12, $e_{1} \in b l c_{h}\left(e_{2}\right)$.

In the opposite direction, $e_{1} \in b l c_{h}\left(e_{2}\right)$ implies, by the second part of $C 1$, that there is a $t^{\prime} \in M C(h)$ such that $e_{2} \in t^{\prime}$ and $e_{2}=\inf \left(t^{\prime \geqslant e_{1}}\right)$. (Definition 12 then assures us that $e_{2} \in f l c_{h}\left(e_{1}\right)$.)

An acquaintance with Minkowski spacetime suggests another requirement on light-cones, which we may call a betweenness property:

Condition 17 ( $C 2$ : betweenness property) Let $h$ be a history and $x, e \in h$.

Then: If $x \in b l c_{h}(e)$, then $\forall y\left(x<y<e \rightarrow y \in b l c_{h}(e)\right)$.

And, if $e \in f l c_{h}(x)$, then $\forall y\left(x<y<e \rightarrow y \in f l c_{h}(x)\right)$.

Condition $C 2$ entails that if $x \in b l c_{h}(e)$ and $y \in b l c_{h}(e)$, then for every $z$ between $x$ and $y, z \in b l c_{h}(e)$. Clearly, if $z=x$ or $z=y$, or $z=e$, then $z \in b l c_{h}(e)$. If neither of the above, since by Fact 13 we have $y \leqslant e$, hence $x<z<e$, so by $C 2: z \in b l c_{h}(e)$. An analogous property holds for $f c_{h}$.

Condition $C 2$ is independent of the BST axioms, which is shown by the BST model exhibited in Fig. 3. Every maximal path such as $t^{\prime}$ that passes through $y$ has a segment above $y$ that is below $e$, which proves that $y \notin b l c_{h}(e)$. On the other hand, path $t$ passing through $x$ guarantees that $x \in b l c_{h}(e)$.

The BST axioms together with conditions $C 1$ and $C 2$ do not guarantee that there are maximal chains passing through an event that go only through the "interior" of the event's light-cones. To illustrate, Fig. 2 shows a one-history BST model, which is the $\mathbb{R}^{2}$ plane, with the shaded area on the right removed. (The borders, however, are in the history.) The ordering is Minkowskian. In this model every maximal chain 


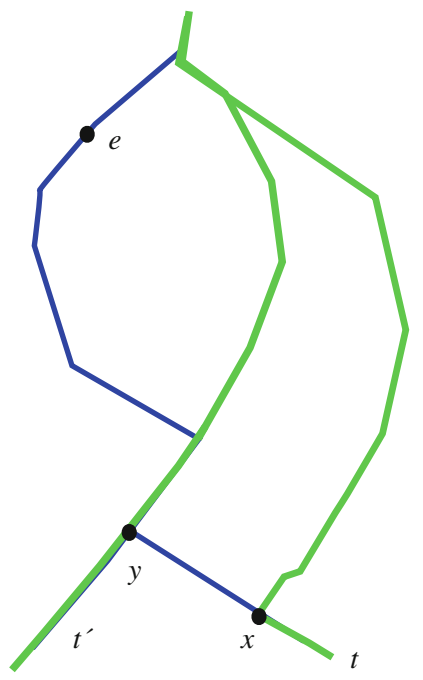

Fig. 3 A pathological one-history BST model, the ordered elements being linked by a line, with the convention that point $x$ not higher on a line than point $y$ means $x \leqslant y$

passing through $e_{1}$ stays for some time on the surface of the future light-cone of $e_{1}$. Condition $C 3$ below prohibits such models.

Condition 18 (C3: interior of light-cones) Let $h \in$ Hist. Then

$\forall e \in h \exists t \in M C(h)\left(e \in t \wedge t^{<e} \neq \varnothing \wedge t^{<e} \cap b l c_{h}(e)=\varnothing \wedge t^{>e} \cap f l c_{h}(e)=\varnothing\right)$.

Note that we need the condition " $t<e \neq \varnothing$ " above since our history might have minimal elements; by the BST axiom of no terminal elements, however, we do not need an analogous condition on $t^{>e}$.

The final condition here considered concerns suprema (infima) of upper (lower) bounded chains in future (backward) light-cones: Such suprema (infima) should belong to future (backward) light-cones.

Condition 19 (C4: limits in light-cones) For every upper bounded chain $C \subseteq$ $f l c_{h}(e)$, where $h \in$ Hist and $e \in W: \sup _{h}(C) \in f l c_{h}(e)$, and for every lower bounded chain $C \subseteq$ blc $_{h}(e)$, where $h \in$ Hist and $e \in W: \inf (C) \in b_{l} c_{h}(e)$.

It is no surprise that the conditions $C 1-C 4$ still permit strange or pathological BST models, such as a real half-plane above (and including) the diagonal $x=y$, with Minkowskian ordering (this is a one-history model). We say that such histories have brims. We do not in this paper attempt to give a condition prohibiting brims. But we aim to give a definition of brims, and put a simple fact relating brims and light-cones. We distinguish two varieties of a history's brim, lower and upper. The intuition underlying our definition is this: If an event $e$ lies on the upper (lower) brim, every maximal chain passing through $e$ has to go along the same path above (below) $e$ - the brim-for some period of time.

Definition 20 (brims) $e$ lies on an upper brim of history $h, e \in u b_{h}$, iff $\exists y(e<y \wedge \forall t \in M C(h)(e \in t \rightarrow \forall x(e \leqslant x \leqslant y \rightarrow x \in t)))$. And $e$ lies on a lower 
brim of history $h, e \in l b_{h}$, iff $\exists y(y<e \wedge \forall t \in M C(h)(e \in t \rightarrow \forall x(y \leqslant x \leqslant$ $e \rightarrow x \in t)))$.

Fact 21 (about brims) For $e, e_{2} \in h$, if $e \in u b_{h}$ and $e<e_{2}$, then $e \notin b l c_{h}\left(e_{2}\right)$. Also, for $e, e_{1} \in h$, if $e \in l b_{h}$ and $e_{1}<e$, then $e \notin f l c_{h}\left(e_{1}\right)$.

Proof For the first assertion, let $e, e_{2}$ be as in the premise and assume for reductio that $e \in b l c_{h}\left(e_{2}\right)$, which entails that for some $t \in M C(h), e \in t$ and $(*) e=\sup _{h}\left(t^{\leqslant e_{2}}\right)$. On the other hand, $e \in u b_{h}$ means that there is a $y$ such that $e<y$ and $\forall t^{\prime}\left(e \in t^{\prime} \rightarrow \forall x\left(e \leqslant x \leqslant y \rightarrow x \in t^{\prime}\right)\right)$. Hence $y \in t$ and also $y, e, e_{2}$ are comparable. If $e<y \leqslant e_{2}$, then $e \neq \sup _{h}\left(t^{\leqslant e_{2}}\right)$, contradicting $(*)$. On the other hand, if $e<y$ and $e_{2}<y$, then $e_{2} \in t$, and hence $\sup _{h}\left(t^{\leqslant e_{2}}\right)=e_{2}$, again contradicting $(*)$, since $e<e_{2}$ by the assumption. The second assertion is proved analogously.

Note that the proof above does not appeal to any of the conditions $C 1-C 4$, which makes one wonder how the existence of brims is related to condition $C 3$. After all, $C 3$ prohibits that every path going through event $e$ cross the surface of the future (past) light-cone of $e$, whereas a brim like diagonal $x=y$ seems to allow for exactly this. That is, we are tempted to read an event $e_{2}$ located on the brim and above $e$ as belonging to the future light-cone of $e$. The fact above orders us to resist this temptation, as such an $e_{2}$ does not belong to $f c_{h}(e)$.

As the final topic related to light-cones, we next prove that although by Definition 12 the relations of belonging to a future light-cone and of belonging to a backward light-cone appear to depend on the history $h$, in fact each is history-independent.

Fact 22 (History-independence) (1) If $e_{2} \in f l c_{h}\left(e_{1}\right)$, then for all $h^{\prime} \in$ Hist containing $e_{1}$ and $e_{2}, e_{2} \in f l c_{h^{\prime}}\left(e_{1}\right)$.

(2) If $e_{1} \in$ blc $c_{h}\left(e_{2}\right)$, then for all $h^{\prime} \in$ Hist containing $e_{1}$ and $e_{2}, e_{1} \in$ blc $_{h^{\prime}}\left(e_{2}\right)$.

Proof Ad 1. For some $t \in M C(h):(*) e_{2}=\inf \left(t^{\geqslant e_{1}}\right)$. Pick an arbitrary $h^{\prime} \in$ Hist such that $e_{1}, e_{2} \in h^{\prime}$. There is thus $t^{\prime} \in M C\left(h^{\prime}\right)$ such that $e_{2} \in t^{\prime}$ and $t^{\leqslant e_{2}}=t^{\prime \leqslant e_{2}}$. If $z \in t^{\prime}$ and $z<e_{2}$, then $z \in t$, and by $(*) z \ngtr e_{1}$, whence $z \notin t^{\prime \geqslant e_{1}}$. By contraposition, if $z \in t^{\prime \geqslant e_{1}}$, then $z /<e_{2}$, and hence $e_{2} \leqslant z$, so $e_{2}$ lower bounds $t^{\prime \geqslant e_{1}}$. Since $e_{2} \in t^{\prime \geqslant e_{1}}$ (by Fact $13 e_{1} \leqslant e_{2}$ ), we get $e_{2}=\inf \left(t^{\prime \geqslant e_{1}}\right)$ and hence $e_{2} \in f l c_{h^{\prime}}\left(e_{1}\right)$.

Ad 2. Assume that $e_{1} \in b l c_{h}\left(e_{2}\right)$; hence $e_{1}=\sup _{h}\left(t^{\leqslant e_{2}}\right)$ for some $t \in M C(h)$. By Fact $13, e_{1} \leqslant e_{2}$. If $e_{1}=e_{2}$, then $e_{1} \in b l c_{h^{\prime}}\left(e_{2}\right)$ because every event lies on its own backward light-cone (in every history containing it). Suppose instead that $e_{1}<e_{2}$. We claim (†) $t^{>e_{1}} \cap h^{\prime} \neq \varnothing$. Suppose otherwise; then $t^{>e_{1}} \subseteq h \backslash h^{\prime}$. So by PCP there is some $c$ such that $c<t^{>e_{1}}$ and $h \perp_{c} h^{\prime}$; but $c<t^{>e_{1}} \operatorname{implies} c \leqslant \inf \left(t^{>} e_{1}\right)=e_{1}<e_{2} \in$ $h \cap h^{\prime}$, contradicting $h \perp_{c} h^{\prime}$. Thus $(\dagger)$. Therefore there are $z \in t^{>e_{1}} \cap h^{\prime}$ and $t^{\prime} \in$ $M C\left(h^{\prime}\right)$ such that $t^{\leqslant z}=t^{\prime \leqslant z}$. Note that $e_{1} \in t^{\leqslant z}=t^{\prime \leqslant z}$ and so $e_{1} \in t^{\prime \leqslant e_{2}}$. We then claim $(\ddagger) t^{\prime \leqslant e_{2}} \leqslant e_{1}$. Fix any $x \in t^{\prime \leqslant e_{2}}$. If $z<x$ then $z<e_{2}$ and so $z \in t^{\leqslant e_{2}}$, which implies $z \leqslant \sup _{h}\left(t^{\leqslant e_{2}}\right)=e_{1}$, contradicting $z \in t^{>e_{1}}$; thus $z /<x$, and $x \leqslant z$ since $z, x \in t^{\prime}$. Hence $x \in t^{\prime \leqslant z}=t^{\leqslant z}$, while $x \in t^{\prime \leqslant e_{2}}$; so $x \in t^{\leqslant e_{2}}$ and hence $x \leqslant$ $\sup _{h}\left(t^{\leqslant e_{2}}\right)=e_{1}$. Therefore $(\ddagger)$, and so $e_{1} \in t^{\prime \leqslant e_{2}}$ is the largest element of $t^{\prime \leqslant e_{2}}$, which implies $e_{1}=\sup _{h^{\prime}}\left(t^{\prime \leqslant e_{2}}\right)$ since $e_{1} \in h^{\prime}$. Thus $e_{1} \in$ blc $_{h^{\prime}}\left(e_{2}\right)$. 
To summarize this section, the axioms of BST were intended only to characterize indeterminism in the sense of allowing events that admit multiple possible historical continuations. As such, they tell us little about individual histories. In particular, the axioms alone do not impose the structure of light-cones on a BST history. In contrast, the conditions $C 1-C 4$ concern individual histories and ensure that $f c_{h}(e)$ and $b l c_{h}(e)$ of Definition 12 behave like future light-cones and backward light-cones of a physical spacetime. Further, $f c_{h}(e)$ and $b l c_{h}(e)$ are historyindependent. This means that our definitions properly generalize the concept of light-cones from a non-modal context of single space-time (history) to the modal context of branching possible histories.

In the sections to follow we will put the four conditions to topological work. In Theorem 35, we will see that these conditions suffice for establishing that each single history has the Hausdorff property, given the topology, $\mathfrak{I}(h)$, per Definition 5. Furthermore, Theorems 44 and 47 link the satisfaction of the Hausdorff property in a many-history BST model with the conditions as well as with passive indeterminism (cf. Definition 39).

\subsection{Borderless Diamonds}

We are about to introduce borderless diamonds, and prove that such objects are open sets of $h$ in the topology $\mathfrak{I}(h)$. (In general, they are not open sets in the topology $\mathfrak{I}(W)$-cf. Fact 6.) We will prove that if a history $h$ satisfies the conditions $C 1-C 4$ of Sect. 3.2, the set of borderless diamonds of $h$ forms a base for topology $\mathfrak{I}(h)$. But first the definition:

Definition 23 (borderless diamonds) $\quad b d^{e_{1} e_{2}} \subseteq h$ is a borderless diamond in history $h, b d^{e_{1} e_{2}} \in B D_{h}$, iff there is a diamond $d^{e_{1} e_{2}} \subseteq h$ such that $b d^{e_{1} e_{2}}=d^{e_{1} e_{2}} \backslash\left(b l c_{h}\left(e_{2}\right)\right.$ $\left.\cup f l c_{h}\left(e_{1}\right)\right)$.

If we want to stress that vertices $e_{1}, e_{2}$ belong to a certain maximal chain $t$ in $h$, we will write $b d_{t}^{e_{1} e_{2}}\left(=b d^{e_{1} e_{2}}\right)$.

Borderless diamonds are open subsets of histories that contain them, which follows from Lemmas 24 and 25 below. They involve $\nabla(x)$ and $\triangle(x)$, that is, the future and past (without brims) of $x$.

Lemma 24 For any $x \in W$, let

$$
\nabla(x):=\left\{z \in W \mid z>x \wedge z \notin f l c_{h}(x)\right\} .
$$

If history $h$ satisfies conditions $C 2$ and $C 4$, then $\nabla(x) \cap h \in \mathfrak{I}(h)$.

Proof If $x \notin h$ then $\nabla(x) \cap h=\varnothing \in \mathfrak{I}(h)$; so let us assume $x \in h$. Let us pick an arbitrary $e \in \nabla(x) \cap h$ and some $t \in M C(h)$ such that $e \in t$. We need to produce an oriented diamond $d_{t}^{x^{*} y^{*}} \subseteq \nabla(x) \cap h$ such that $e \in d_{t}^{x^{*} y^{*}}$. To find a bottom vertex $x^{*}$, consider $t^{\geqslant x} \cap f l c_{h}(x)$. Observe first that inf $\left(t^{\geqslant x}\right)$ is well-defined since $e \in t^{\geqslant x}$ and $x \leqslant t^{\geqslant x}$. Since $\inf \left(t^{\geqslant x}\right) \geqslant x$ and $\inf \left(t^{\geqslant x}\right) \in f l c_{h}(x), t^{\geqslant x} \cap f l c_{h}(x)$ is non-empty. It is also upper bounded by $e$ since $e$ is comparable with every $z \in t^{\geqslant x} \cap f l c_{h}(x)$ and 
$e<z$ contradicts $C 2$, as $z \in f l c_{h}(x)$ but $e \notin f l c_{h}(x)$. Accordingly $t^{\geqslant x} \cap f l c_{h}(x)$ has a supremum-in- $h, s=\sup _{h}\left(t^{\geqslant x} \cap f l c_{h}(x)\right)$, and $s \in t$. By $C 4, s \in f l c_{h}(x)$. Moreover, $s<e$; otherwise $e \in f l c_{h}(x)$, which is prohibited by the second conjunct of the definition of $\nabla(x)$. By density, we pick some $x^{*} \in t^{\geqslant x}$ such that $s<x^{*}<e$. Hence $x^{*} \notin f l c_{h}(x)$. Moreover, for every $z \in h$, if $z \geqslant x^{*}$, then $z>x$ and $z \notin f l c_{h}(x)$ (by $C 2$ ), so $z \in \nabla(x)$. Accordingly, for every $y^{*} \in t^{>e} \subseteq h, d_{t}^{x^{*} y^{*}} \subseteq \nabla(x) \cap h$.

Lemma 25 For any $x \in W$, let

$$
\triangle(x):=\left\{z \in W \mid z<x \wedge z \notin b l c_{h}(x) \wedge z \text { is not minimal in } W\right\} .
$$

If history $h$ satisfies conditions $C 2$ and $C 4$ and moreover $x \in h$ (which implies $\triangle(x) \subseteq h$ and $\triangle(x) \cap h=\triangle(x))$, then $\triangle(x) \in \mathfrak{I}(h)$.

Proof We proceed similarly to the proof for Lemma 24, picking an arbitrary $e \in \triangle(y)$ and some $t \in M C(h), e \in t$, and then looking for $d_{t}^{x^{*} y^{*}}$, with $e \in d_{t}^{x^{*} y^{*}}$, this time concentrating on its top vertex $y^{*} \in t^{\leqslant y}$. We consider $t^{\leqslant y} \cap b l c_{h}(y)$. Note that $\sup _{h}\left(t^{\leqslant y}\right)$ is well-defined since $e \in t^{\leqslant y}$ and $y \geqslant t^{\leqslant y}$. Since $\sup _{h}\left(t^{\leqslant y}\right) \leqslant y$ and $\sup _{h}\left(t^{\leqslant y}\right) \in b l c_{h}(y), t^{\leqslant y} \cap b l c_{h}(y)$ is non-empty; it is also lower bounded by $e$ since for every $z \in t^{\leqslant y} \cap b c_{h}(y), e$ and $z$ are comparable, and $z<e$ contradicts $C 2$. Accordingly there is an infimum $f=\inf \left(t^{\leqslant y} \cap b l c_{h}(y)\right)$ and $f \in t$. By $C 4$, $f \in b l c_{h}(y)$. Moreover, $e<f$; otherwise $e \in b l c_{h}(y)$. By density, there is some $y^{*} \in$ $t^{\leqslant y}$ such that $e<y^{*}<f$. Hence $y^{*} \notin b l c_{h}(y)$. Moreover, for every $z \in h$, if $z \leqslant y^{*}$, then $z<y$ and $z \notin b l c_{h}(y)$ (by $C 2$ ), so $z \in \triangle(y)$. Thus, for every $x^{*} \in t^{<e}$ that is not minimal in $W$ (which exists since $e \in \triangle(y)$ is not minimal in $W$ ), $d_{t}^{x^{*} y^{*}} \subseteq \triangle(y)$.

Note that $\triangle(x)$ is simply $\left\{z \in W \mid z<x \wedge z \notin b l c_{h}(x)\right\}$ if $W$ satisfies $C 3$ (or, in fact, if $W$ has no minimal elements).

Lemma 26 Let history $h$ satisfy conditions $C 2$ and $C 4$. Then the borderless diamonds on $h$ are open sets of $h$, that is, $B D_{h} \subseteq \mathfrak{I}(h)$.

Proof Fix any $b d^{x y} \in B D_{h}$. No $e \in b d^{x y}$ is minimal in $W$ since $x<b d^{x y}$. So, because $b d^{x y} \subseteq h$, it is the intersection of $\nabla(x) \cap h$ and $\triangle(y)$, both of which are in $\mathfrak{I}(h)$ by Lemmas 24 and 25 . Hence $b d^{x y} \in \mathfrak{I}(h)$.

We have learned from Lemma 26 that borderless diamonds are particularly simple open sets of history $h$. Do they form a base for topology $\mathfrak{I}(h)$ ? The following lemma suffices.

Lemma 27 Let a history $h$ satisfy conditions $C 1$ and $C 3$ and let $A \in \mathfrak{I}(h)$. Then for every $e \in A$, some borderless diamond contained in A contains e.

Proof By $C 3$ there is $t \in M C(h)$ such that both $t^{<e}$ and $t^{>e}$ are nonempty and $t^{<e} \cap b l c_{h}(e)=\varnothing$ and $t^{>e} \cap f c_{h}(e)=\varnothing$. If $A=h$, pick any $e_{1} \in t^{<e}, e_{2} \in t^{>e}$ and then $e \in d_{t}^{e_{1}, e_{2}} \subseteq h=A$; on the other hand, if $A \neq h$, then the openness of $A$ implies that there are $e_{1} \in t^{<e}$ and $e_{2} \in t^{>e}$ such that $e \in d_{t}^{e_{1}, e_{2}} \subseteq A$. It follows that $e_{1} \notin b l c_{h}(e)$ and $e_{2} \notin f l c_{h}(e)$, and hence $e \notin\left(f l c_{h}\left(e_{1}\right) \cup b l c_{h}\left(e_{2}\right)\right)$ (by $\left.C 1\right)$. Accordingly, $e$ is not on a border of $d_{t}^{e_{1} e_{2}}$, so $e \in b d^{e_{1} e_{2}} \subseteq A$. 
Theorem 28 Let $h \in$ Hist satisfy conditions $C 1, C 2, C 3$, and $C 4$. Then the set $B D_{h}$ of borderless diamonds on $h$ forms a base for the topology $\mathfrak{I}(h)$, that is

$$
\forall A \subseteq h A \in \mathfrak{I}(h) \text { iff } \exists \mathcal{B} \subseteq B D_{h}(\bigcup \mathcal{B}=A) .
$$

Proof By Lemma 26, conditions $C 2$ and $C 4$ guarantee that borderless diamonds are open subsets of $h$. To construct $\mathcal{B} \subseteq B D_{h}$ that has the above property, let us put $\mathcal{B}:=\left\{b d \in B D_{h} \mid b d \subseteq A\right\}$. To prove that $\bigcup \mathcal{B} \subseteq A$, pick $b d \in \mathcal{B}$. Then by the definition of $\mathcal{B}, b d \subseteq A$. Lemma 27 , which requires $C 1$ and $C 3$, gives us the opposite direction.

Theorem 28 also means that if a history $h$ satisfies conditions $C 1$ through $C 4$, the set of $\nabla(x) \cap h$ and $\triangle(y)$ (for $x, y \in h$ ) forms a subbase for $\mathfrak{I}(h)$. It is also worth noting that Lemma 24 has the following corollary.

Corollary $29 \nabla(x) \in \mathfrak{I}(W)$ if $W$ satisfies conditions $C 2$ and $C 4$.

Proof From Lemma 24 by Theorem 7.

We should note that, in contrast, Lemma 25 (which needs the assumption that $x \in h$ ) fails to entail the $\triangle(x)$ counterpart of this corollary, in particular, by Fact 6 , when $\triangle(x)$ contains a choice point.

\section{Hausdorff Property on a Single Spacetime (History)}

We next have a theorem relevant to the critical question whether a single spacetime (BST history) has the Hausdorff property. We prove the theorem with two little lemmas in addition to Lemma 24. The chief idea is to separate two distinct points into $\nabla(x)$ and $M(x)$, the regions inside and outside the forward light-cone of some point $x$, which is possible due to Fact 33 .

Fact 30 For any $e_{1}, e_{2} \in W$ and $t \in M C(W)$ with $e_{2} \in t$, if $e_{1} \nless e_{2}$ then $e_{1} \nless x$ for some $x \in t_{2}>e_{2}$.

Proof If $e_{1} \leqslant x$ for all $x \in t_{2}>e_{2}$, then $e_{1} \leqslant \inf \left(t_{2}>e_{2}\right)=e_{2}$.

Lemma 31 For any $x \in W$, let

$$
\mathrm{M}(x):=\{e \in W \mid x \nless e \wedge e \text { is not minimal in } \mathrm{W}\} .
$$

Then $\triangle(x) \cap h \in \mathfrak{I}(h)$ for any history $h$.

Proof Fix any $e \in \mathrm{M}(x) \bigcap h$ and $t \in M C(h)$ such that $e \in t$. Then Fact 30 implies $x \nless z y$ for some $y \in t^{>e}$; moreover, $x \nless z \in h$ for any $z \leqslant y$ (since $y \in t \subseteq h$ ). Therefore, for any $z \in t^{<e}$ that is not minimal in $W$ (which exists since $e$ is not minimal in $W$ ), we have $z<e<y$ and $d^{z y} \subseteq \square(x) \bigcap h$. 
Note that $M(x)$ is simply $\{z \in W \mid z \nsupseteq x\}$ if $W$ satisfies $C 3$ (or, in fact, if $W$ has no minimal elements). Note also that, like Corollary 29, Lemma 31 gives an open set of $W$ :

Corollary $32 M(x) \in \mathfrak{I}(W)$.

Proof From Lemma 31 by Theorem 7.

The following fact is a crucial element for the Hausdorff property of a history. It is symmetrical to Fact 30 within a history but, unlike Fact 30, does not extend to Our World (without a pathological assumption).

Fact 33 For any history $h, e_{1}, e_{2} \in h$, and $t \in M C(h)$ with $e_{1} \in t$ and $t^{<e_{1}} \neq \varnothing$, if $e_{1} \nless e_{2}$ then $x \notin e_{2}$ for some $x \in t^{<e_{1}}$.

Proof If $x \leqslant e_{2}$ for all $x \in t^{<e_{1}}$, then $e_{1}=\sup _{h}\left(t^{<e_{1}}\right) \leqslant e_{2}$.

Lemma 34 Suppose that a history $h$ satisfies $C 3$ and that $e_{1} \nless e_{2}$ for $e_{1}, e_{2} \in h$. Then there is some $x \in h$ such that $x \in \triangle\left(e_{1}\right)$ (which implies $e_{1} \in \nabla(x)$ if h satisfies $C 1)$ and $e_{2} \in M(x)$.

Proof Applying C3, pick $t \in M C(h)$ such that $e_{1} \in t$ and $t^{<e_{1}} \neq \varnothing$ but $t^{<e_{1}} \cap b_{b} c_{h}\left(e_{1}\right)=\varnothing$. Then by Fact 33 there is $x \in t^{<e_{1}}$ such that $x \notin e_{2}$, that is, $e_{2} \in M(x)$, and moreover $x \in \triangle\left(e_{1}\right)$ since $t^{<e_{1}} \cap b l c_{h}\left(e_{1}\right)=\varnothing$.

Theorem 35 Let history h of a BST model OW satisfy conditions $C 1, C 2, C 3$, and $C 4$. Then $h$ satisfies the Hausdorff property (in the topology $\mathfrak{I}(h)$ introduced in Definition 5).

Proof Pick any distinct $e_{1}, e_{2} \in h$; because $e_{1} \leqslant e_{2}$ and $e_{2} \leqslant e_{1}$ would entail $e_{1}=e_{2}$, we may assume without loss of generality that $e_{1} \nless e_{2}$. By Lemma 34,C1 and $C 3$ imply that $e_{1} \in \nabla(x) \cap h$ and $e_{2} M(x) \cap h$ for some $x \in h$, where $(\nabla(x) \cap$ $h) \cap(\triangle(x) \cap h)=\varnothing$ by definition. $\triangle(x) \cap h \in \mathfrak{I}(h)$ by Lemma 31, whereas $C 2$ and $C 4$ entail $\nabla(x) \cap h \in \mathfrak{I}(h)$ by Lemma 24 .

It may be worth noting that Theorem 35 can also be proved with $\triangle(x)$ and $\emptyset(x)$ as in the following lemma (which, however, does not extend to Our World in the way Lemma 31 extends to Corollary 32).

Lemma 36 For any $x \in W$, let

$$
\varpi(x):=\{e \in W \mid e \not z x \wedge e \text { is not minimal in } \mathrm{W}\} .
$$

Then $\emptyset(x) \cap h \in \mathfrak{I}(h)$ for any history $h$ such that $x \in h$.

Proof Fix any $e \in \emptyset(x) \bigcap h$ and $t \in M C(h)$ such that $e \in t$. Since $e$ is not minimal, $t^{<e} \neq \varnothing$. So Fact 33 implies $z \not x$ for some $z \in t^{<e}$; moreover, for any $y \geqslant z, y \notin x$ and $y$ is not minimal. Without loss of generality we may assume that $z$ is not minimal (since if it is we can find some $z^{\prime} \in t$ such that $z<z^{\prime}<e$ and hence $z^{\prime} \nless x$ and $z^{\prime}$ is not minimal). Therefore, for any $y \in t^{>} e$, we have $z<e<y$ and $d^{z y} \subseteq \emptyset(x)$. 
To comment on the results of this section, each single history $h$ (aka space-time) of a BST model satisfies the Hausdorff property with respect to the Bartha topology $\mathfrak{I}(h)$, provided that $h$ satisfies conditions $C 1-C 4$. In turn, these conditions are needed in order that a light-cone in a BST history have some typical properties required of light-cones of space-time physics. This ultimately responds to an objections voiced by Earman (2008) that space-times of BST are non-Hausdorff.

\section{Hausdorff Property on Our World}

In this section we are after a completely different game: Instead of focusing on single histories, we ask about a topological feature of Our World of many branching histories.

To present our next theorem, we need to introduce a particular feature of BST, called "passive indeterminism," formerly labeled "indeterminism without choice" (cf. Belnap 1992) or "indeterminism with external choice." It is impossible to be both brief and precise. The rough intuitive idea, spelled out in detail in Belnap (2005), is that there is a single initial event, I, serving as the common initial of multiple contingent transitions, each with its own event, $O_{j}$, as possible outcome. I must lie in the causal past of each $O_{j}$. If $\mathbf{I}$ is a point event, $e$, and if $O$ is an immediate outcome of $e$ (no intervening events), then the transition $e \rightarrow O$ is a causa causans or originating cause that is not subject to further causal analysis. A causa causans might well be labeled as a case of active indeterminism. In the more typical case, a contingent transition, $\mathbf{I} \rightarrow O$ is not a causa causans; instead, it has its own set of causae causantes external to it that provably form a set of inus conditions in the sense of Mackie (1974): Each is an insufficient but nonredundant (conjunctive) part of an unnecessary but sufficient condition of $\mathbf{I} \rightarrow O$. Such an $\mathbf{I} \rightarrow O$ illustrates passive indeterminism, since the activity resides in its causae causantes, the initials of which always lie in the past of $O$. For example, suppose you have already placed your bet on the (future) decay of an $\alpha$-particle. It may be that the transition from bet-placed to bet-won is indeterministic, but what causes the win (if you win) is, say, the detection of the decay of the $\alpha$-particle.

Passive indeterminism can be cleanly characterized by considering various ways in which two maximal chains $t_{1}, t_{2} \in M C(W)$ can form a $Y$-shaped fork.

Definition 37 (Y-fork) $t_{1}, t_{2} \in M C(W)$ form a Y-fork iff

1. $t_{1} \neq t_{2}$,

2. $t_{1} \cap t_{2} \neq \varnothing$,

3. $\forall x \in t_{1} \cup t_{2}\left(x \in t_{1} \cap t_{2} \vee t_{1} \cap t_{2}<x\right)$.

Every Y-fork has its trunk $t_{1} \cap t_{2}$, and two arms $t_{1} \backslash t_{2}$ and $t_{2} \backslash t_{1}$. A Y-fork may be entirely a spatio-temporal matter. In order to characterize Y-forks that exhibit indeterminism, we define a "modal fork" as follows.

Definition 38 (modal-fork) $t_{1}, t_{2} \in M C(W)$ form a modal fork iff they form a Y-fork and $\exists h_{1}, h_{2} \in \operatorname{Hist}\left(t_{1} \backslash t_{2} \subseteq h_{1} \backslash h_{2} \wedge t_{2} \backslash t_{1} \subseteq h_{2} \backslash h_{1}\right)$. 
In the presence of Definition 37, the condition on modal forks is equivalent to this claim:

$$
\forall x \in t_{1} \cup t_{2}\left(t_{1} \cap t_{2}<x \rightarrow x \in h_{1} \backslash h_{2} \vee x \in h_{2} \backslash h_{1}\right) .
$$

There is a simple topological test for whether or not a certain modal Y-fork exhibits passive indeterminism. If its trunk, $t_{1} \cap t_{2}$, has a (unique) maximum, $e$, then $e$ is the initial of two causae causantes, the left fork being one possible outcome, and the right fork the other, and the indeterminism may be called "active." If, however, the trunk has no maximum, the Y-fork exhibits passive indeterminism, and we must look elsewhere for the relevant causae causantes (cf. Belnap 2005). For our topological purposes, we convert this test into a definition.

Definition 39 (passive indeterminism) A modal fork exhibits passive indeterminism in case its trunk has no maximum.

The upshot of these definitions is a threefold classification of Y-forks. A Y-fork may be modal or not, and if is modal, it either exhibits passive indeterminism or not. Note that the essence of passive indeterminism is not a matter of lacking a choice event (this is impossible by the prior choice principle of BST), but that every choice event is external to the modal fork in question. The lemma that follows the fact below shows a crucial feature of passive indeterminism:

Fact 40 (1) Let $t_{1}, t_{2} \in M C(W)$ form a $Y$-fork. Then its trunk is upper bounded by an element of each of $t_{1} \backslash t_{2}$ and $t_{2} \backslash t_{1}$. (2) And, if $t_{1}, t_{2}$ form a modal fork, i.e., for some $h_{1}, h_{2} \in$ Hist, $t_{2} \backslash t_{1} \subseteq h_{1} \backslash h_{2}$ and $t_{2} \backslash t_{1} \subseteq h_{2} \backslash h_{1}$, then $\forall x \in t_{1} \cup t_{2} \quad\left(x \in h_{1} \cap\right.$ $\left.h_{2} \rightarrow x \in t_{1} \cap t_{2}\right)$.

Proof Ad 1. By Definition 37(1) and maximality of $t_{1}, t_{2}$, there is $x \in t_{1} \backslash t_{2}$, so $x \notin t_{1} \cap t_{2}$, so $t_{1} \cap t_{2}<x$ by Definition 37(3); and similarly for $t_{2} \backslash t_{1}$. Ad 2. The result follows by observing that $t_{1} \cup t_{2}=\left(t_{1} \cap t_{2}\right) \cup\left(t_{1} \backslash t_{2}\right) \cup\left(t_{2} \backslash t_{1}\right)$.

Lemma 41 The following two claims are equivalent, for $t_{1}, t_{2} \in M C(W)$ :

1. $t_{1}, t_{2}$ form a modal fork and $t_{1} \cap t_{2}$ has no maximum;

2. there exist $\sup _{h_{1}}\left(t_{1} \cap t_{2}\right)$ and $\sup _{h_{2}}\left(t_{1} \cap t_{2}\right)$ and $\sup _{h_{1}}\left(t_{1} \cap t_{2}\right) \neq \sup _{h_{2}}\left(t_{1} \cap t_{2}\right)$ for some $h_{1}, h_{2} \in$ Hist.

Proof From (1) to (2). Let us abbreviate $\Gamma=\left(t_{1} \cap t_{2}\right), \gamma_{1}=\sup _{h_{1}}(\Gamma), \Delta_{1}=$ $\left(t_{1} \backslash t_{2}\right)$, and similarly for $\gamma_{2}, \Delta_{2}$. We show that $\gamma_{1} \neq \gamma_{2}$.

Note that $\left(\Gamma \cup \Delta_{1}\right)=t_{1}$ by calculation, $\Gamma \neq \varnothing$ by Definition $37(2), \Delta_{1} \neq \varnothing$ by Fact 40, and $\Gamma<\Delta_{1}$ by Definition 37(3). By Definition 38, $\Delta_{1} \subseteq h_{1} \backslash h_{2}$, so $\Gamma \subseteq h_{1}$ by downward closure of histories, so $\gamma_{1}$ exists (by the BST axiom of history-relative suprema) and $\Gamma \leqslant \gamma_{1} \leqslant \Delta_{1}$. Since $\left(\Gamma \cup \Delta_{1}\right)=t_{1}$, density and maximality of $t_{1}$ imply that $\gamma_{1} \in\left(\Gamma \cup \Delta_{1}\right)$. If $\gamma_{1} \in \Gamma$, then $\gamma_{1}$ would be maximum in $\Gamma$, violating assumption (1) of the Lemma. So $\gamma_{1} \in \Delta_{1}$. An exactly parallel argument yields $\gamma_{2} \in \Delta_{2}$. Since $\left(\Delta_{1} \cap \Delta_{2}\right)=\varnothing, \gamma_{1} \neq \gamma_{2}$. From (2) to (1). $\sup _{h_{1}}\left(t_{1} \cap t_{2}\right) \neq \sup _{h_{2}}\left(t_{1} \cap t_{2}\right)$ implies that $t_{1} \cap t_{2}$ has no maximum. It is straightforward to see that $t_{1}, t_{2}$ form a modal fork. 
We are now ready to state our first theorem concerning the Hausdorff property in the topology $\mathfrak{I}(W)$.

Theorem 42 Let $O W$ be a BST model. If some modal fork in OW exhibits passive indeterminism, then the Hausdorff property fails in $O W$ (in the topology $\mathfrak{I}(W)$ ).

Proof Let $t_{1}, t_{2} \in M C(W)$ form a modal fork exhibiting passive indeterminism. Then by Lemma 41 there are histories $h_{1}, h_{2}$ such that $t_{1} \cap t_{2} \subseteq h_{1} \cap h_{2}$ and $e_{1}:=\sup _{h_{1}}\left(t_{1} \cap t_{2}\right) \neq \sup _{h_{2}}\left(t_{1} \cap t_{2}\right)=: e_{2}$. Let $e_{1} \in U$ and $e_{2} \in V$, where $U$ and $V$ are arbitrary open subsets of $W$. Since $U$ is open, there must be $x_{1} \in t_{1} \cap t_{2}$ such that $x_{1}<e_{1}$ and $\forall x\left(x_{1} \leqslant x \leqslant e_{1} \rightarrow x \in U\right)$. For a similar reason there is $x_{2} \in t_{1} \cap t_{2}$ such that $x_{2}<e_{2}$ and $\forall x\left(x_{2} \leqslant x \leqslant e_{2} \rightarrow x \in V\right)$. Thus, $\max \left(x_{1}, x_{2}\right) \in U \cap V$.

A natural question, answered by the lemma below, is, How ubiquitous is passive indeterminism ${ }^{13}$

Lemma 43 Let $e$ be a choice point between histories $h$ and $h^{\prime}$ (to be written as $\left.e \in C P\left(h, h^{\prime}\right)\right)$ in a BST model and for some $e^{\prime} \neq e, \quad e^{\prime} \in f l c_{h}(e)$ and $\forall c\left(c \in C P\left(h, h^{\prime}\right) \wedge c<e^{\prime} \rightarrow c=e\right)$. Then there is passive indeterminism in the model.

Proof Clearly, $e, e^{\prime} \in h$ and by Fact 13 (1) and the antecedent, $e<e^{\prime}$. Also ( $\dagger$ ) $e^{\prime} \notin h^{\prime}$-otherwise $e$ would not be a choice point for $h$ and $h^{\prime} . e^{\prime} \in f l c_{h}(e)$ entails that $(\ddagger) \exists t \in M C(h)\left(e^{\prime}=\inf \left(t^{\geqslant e}\right)\right)$. Consider $t^{\ngtr e}$. Since $e \leqslant t^{\geqslant e}, e \neq \inf \left(t^{\geqslant e}\right)$, and $t$ is maximal, $t^{\ngtr e} \neq \varnothing$. Since it is upper bounded by $e^{\prime}$, it has a supremum and $\sup _{h}\left(t^{\ngtr e}\right)=e^{\prime}$ (by $\ddagger$ ). We next argue that $t^{\ngtr e} \subseteq h^{\prime}$. Otherwise for some $e^{*} \in t^{\ngtr e}$, $e^{*} \notin h^{\prime}$ and hence $e^{*} \in h \backslash h^{\prime}$, so by PCP, $\exists c \in C P\left(h, h^{\prime}\right)\left(c<e^{*}\right)$. But because $e^{*} \in t^{\ngtr e}, e /<e^{*}$. Hence $c \neq e$ and since $c<e^{*} \leqslant e^{\prime}$, we have a contradiction with the Lemma's premise.

We may thus consider $\sup _{h^{\prime}}\left(t^{\ngtr e}\right)=s$. (†) then requires that $s \neq e^{\prime}$.

We thus constructed a trunk $t^{\ngtr e}$ of a modal fork, with two (different) history-relative suprema. By Lemma 41 and Definition 39, the fork exhibits passive indeterminism.

To comment on this Lemma, putting aside some pathological BST models, if a BST model has more than one history, it exhibits passive indeterminism. The pathology means that either for every choice point $e, f c_{h}(e)=\{e\}$ for any $h$ such that $e \in h,{ }^{14}$ or each choice point between some $h, h^{\prime}$ is a point of convergence of a sequence of choice points between $h$ and $h^{\prime}$. Combining our Theorem 42 and Lemma 43, we obtain the following:

Theorem 44 Let $e \in C P\left(h, h^{\prime}\right)$ for some histories $h, h^{\prime}$ in a BST model $O W$ and for some $e^{\prime} \neq e, e^{\prime} \in f l c_{h}(e)$ and $\forall c \forall_{c}\left(c \in C P\left(h, h^{\prime}\right) \wedge c<e^{\prime} \rightarrow c=e\right)$. Then the Hausdorff property fails in $O W$ (in the topology $\mathfrak{I}(W)$ ).

\footnotetext{
13 The conclusion of this lemma, namely, the existence of passive indeterminism, can be also proved by postulating No Modal Funny Business instead of the lemma's second premise, i.e., instead of $\forall c\left(c \in C P\left(h, h^{\prime}\right) \wedge c<e^{\prime} \rightarrow c=e\right)$. For a definition of No Modal Funny Business, cf. Def. 1-2 of Belnap (2003b).

14 Note that this is satisfied in BT models, so these models do not exhibit passive indeterminism.
} 
It is still interesting to learn what happens if a BST model has no passive indeterminism, which is a subject of Theorem 47. Note however that no passive indeterminism in a BST model means that either the model has one history only, or (by Lemma 43) is pathological.

We can prove Theorem 47 using the same idea that we used to prove Theorem 35 , since Fact 33 carries over to $O W$ in the absence of passive indeterminism.

Fact 45 Suppose no modal fork in $O W$ exhibits passive indeterminism. Then, for any $e_{1}, e_{2} \in W$ and $t \in M C(W)$ such that $e_{1} \in t$ and $t^{<e_{1}} \neq \varnothing$, if $e_{1} \nless e_{2}$ then $x \notin e_{2}$ for some $x \in t^{<e_{1}}$.

Proof Suppose $e_{1} \nless e_{2}$ but that $x \leqslant e_{2}$ for all $x \in t^{<e_{1}}$. Pick any $h_{1} \in H_{\left(e_{1}\right)}$ and $h_{2} \in H_{\left(e_{2}\right)} ;$ moreover, since $t^{<e_{1}} \leqslant e_{2}$, there is $t_{2} \in M C\left(h_{2}\right)$ such that $t^{<e_{1}} \cup\left\{e_{2}\right\} \subseteq t_{2}$. Then $e_{1}=\sup _{h_{1}}\left(t^{<e_{1}}\right)$ whereas $\sup _{h_{2}}\left(t^{<e_{1}}\right) \leqslant e_{2}$; hence $e_{1} \not e_{2}$ implies $\sup _{h_{1}}\left(t^{<e_{1}}\right) \neq \sup _{h_{2}}\left(t^{<e_{1}}\right)$, which means that $e_{1} \notin h_{2}$, which moreover entails $t \cap t_{2}=t^{<e_{1}}$. Therefore, by Lemma 41, the modal fork $t, t_{2}$ exhibits passive indeterminism.

Lemma 46 Suppose no modal fork in OW exhibits passive indeterminism, that $O W$ satisfies $C 3$, and that $e_{1} \nless e_{2}$ for $e_{1}, e_{2} \in W$. Then there is some $x \in W$ such that $x \in \triangle\left(e_{1}\right)$ (which implies $e_{1} \in \nabla(x)$ if $W$ satisfies $C 1$ ) and $e_{2} \in M(x)$.

Proof Similar to the proof of Lemma 34, using Fact 45 in place of Fact 33.

Theorem 47 Let $O W$ be a BST model, in which every history satisfies conditions $C 1, C 2, C 3$, and $C 4$. Then if no modal fork in $O W$ exhibits passive indeterminism, the Hausdorff property is satisfied in $O W$ (in the topology $\mathfrak{I}(W)$ ).

Proof Exactly similar to the proof of Theorem 35. Pick any distinct $e_{1}, e_{2} \in W$; without loss of generality we may assume $e_{1} \nless e_{2}$. By Lemma 46, $C 1$ and $C 3$ imply that $e_{1} \in \nabla(x)$ and $e_{2} \in M(x)$ for some $x \in W$, where $\nabla(x) \cap M(x)=\varnothing$ by definition. $M(x) \in \mathfrak{I}(W)$ by Corollary 32 , whereas $C 2$ and $C 4$ entail $\nabla(x) \in \mathfrak{I}(W)$ by Corollary 29.

The theorems of this section nicely contrast with our results concerning single histories: With a few exceptions, a BST model $\langle W, \leqslant\rangle$ is non-Hausdorff with respect to the Bartha topology on $W$.

\section{Naturalness of the BST Topologies}

All our results above obtain with respect to specific topologies: the Bartha topology $\mathfrak{I}(h)$ on a BST history, and the Bartha topology $\mathfrak{I}(W)$ on a BST model. This naturally raises a question about strength and relevancy of these results. Aren't perhaps the Bartha topologies some gerrymandered and ad hoc constructions, cooked up just to to guarantee that the lemma and theorems above hold? Against this objection in each of the next three subsections we provide an independent argument that the Bartha topologies on a BST model and its histories are natural topologies: 
1. In appropriate cases, the Bartha topology on a single history (spacetime) agrees with the standard topology on $\mathbb{R}^{n}$.

2. Both on a BST model and on a history thereof, the Bartha topology naturally extends the BST structure.

3. The Bartha topology on Our World, an entire BST model, is canonically constructed from the Bartha topologies on its histories.

\subsection{Extending the Minkowski Structure}

One simple criterion of "naturalness" of topology is whether it agrees with the standard "ball" topology on $\mathbb{R}^{n}$, if its base set is isomorphic to $\mathbb{R}^{n}$. It is easy to see that the criterion is satisfied by the Bartha topology on a history, $\mathfrak{I}(h)$. There is a particular class of BST models, called Minkowskian Branching Structures (MBS's) and extensively studied by Müller (2002), Wroński and Placek (2009), and Placek and Belnap (2012), in which histories are isomorphic to $\mathbb{R}^{n}$ with Minkowskian ordering. More specifically, elements of an MBS's base set are certain equivalence classes $[\sigma x]$, where $\sigma \in \Sigma$ for some index set $\Sigma$ and $x \in \mathbb{R}^{n}$, whereas the ordering generalizes the Minkowskian ordering on $\mathbb{R}^{n}$. Each history in an MBS is then identified with set $b_{\sigma}=\left\{[\sigma x] \mid x \in \mathbb{R}^{n}\right\}$ for some $\sigma \in \Sigma$. To inquire what borderless diamonds in history $b_{\sigma}$ are (recall that such diamonds form the base of the Bartha topology on a history, $\mathfrak{I}\left(b_{\sigma}\right)$ ), we get that they have the form:

$$
b d^{[\sigma x][\sigma y]}=\left\{[\sigma z] \in b_{\sigma} \mid x<_{M} z<_{M} y \wedge z \notin f l c(x) \cup b l c(y)\right\},
$$

where $<_{M}$ is the strict companion of the Minkowskian ordering on $\mathbb{R}^{n}$ and $f l c(x), \operatorname{blc}(x)$ are the future light-cone and the backward light-cone of $x \in \mathbb{R}^{n}$. A little reflection on the condition above convinces one that $z$ is an element of the borderless diamond in $\mathbb{R}^{n}$, with $x$ its bottom diamond and $y$ its top diamond. Such borderless diamonds are open in the standard topology on $\mathbb{R}^{n}$. Thus, the base for the Bartha topology on a Minkowskian Branching Structure is provided by the sets of the form $b d^{[\sigma x][\sigma y]}$, whose "numerical" parts are open sets in the standard topology on $\mathbb{R}^{n}$.

\subsection{Topological Limits and BST Limits}

Two notions of limits are involved with BST and topology. One is an intrinsic ingredient of BST, namely, inf and sup. The other is the convergence of "nets" (a generalization of sequences) in topology — any topology gives rise to a convergence relation between nets and points. Indeed, topologies are fully characterized by convergence relations, in the sense that different topologies give different convergence relations, and that any topology can be recovered from the convergence relation it gives. Therefore any natural topology of BST must give a topological limit (that is, a convergence) that extends the BST limit (that is, inf and sup), in the sense that the two kinds of limits agree whenever the latter makes sense. This is in fact the case with the Bartha topology, as in Theorems 49 and 50 below. 
A net is a function from a directed set $(D, \leqslant)$ to a topological space $X .^{15}$ A salient example is a sequence in $X$, that is, a map from $(\mathbb{N}, \leqslant)$ to $X$. The formal concept of convergence of a net is a formalization (and generalization) of the idea that a sequence may "get arbitrarily close" to a point $x \in X$. The notion of closeness involved in this idea is abstractly captured by the topology on $X$. On the other hand, the idea also involves a somewhat temporal aspect: When $f(n)$ is closer to $x$ than $f(m)$ is, whether the sequence $f$ gets closer to or farther from $x$ between the two "stages" $n$ and $m$ hinges on whether $n$ is "after" $m$ or not, which is expressed by the order on the domain $D$ of a net.

The definition of convergence goes as follows. Given a net $f: D \rightarrow X$ and a subset $U \subseteq X$, we say that $f$ "eventually stays" in $U$ if there is $a \in D$ such that $f(b) \in U$ for every $b \geqslant a$. For instance, a sequence $f: \mathbb{N} \rightarrow X$ eventually stays in $U \subseteq X$ if, "after" some stage $n, f$ is always in $U$ (meaning, $f(m) \in U$ for every $m \geqslant n$ ). Given this notion of eventually staying, and given the topological abstraction of "closeness" (points in an open set $U$ containing $x$ are "closer" to $x$ than ones outside $U$ ), the idea of "getting arbitrarily close" is formalized as follows: A net $f$ converges (in the given topology of $X$ ) to a point $x \in X$ if, for every open set $U$ containing $x, f$ eventually stays in $U$ (that is, there is $a \in D$ such that $f(b) \in U$ for every $b \geqslant a)$.

As a particular case, a chain $C$ in a BST model $(W, \leqslant)$ can be regarded as a net by taking the inclusion map $i: C \rightarrow W$, which maps $x \in C$ to $x$ itself. A warning may well be in order, that there is going to be possible confusion, because the same points $x \in C$ are serving once as points of the directed domain (that is, as "stages") of a net, and once as points of spacetimes or of Our World, and because the same ordering $\leqslant$ is used, once as the ordering on the directed domain (the ordering of stages) of the net, and once as the BST ordering (of causal past and future). To avoid confusion, we reserve the temporal phrasing ("after") for when we emphasize the order as the ordering on the domain of a net, and, taking advantage of familiar pictures of Our World, we say that a point $x \in W$ is "below" or "above" $y$ (if $x \leqslant y$ or $y \leqslant x$ ) to emphasize the order as the BST ordering.

A chain $C$ in a BST model $(W, \leqslant)$ can be regarded as an "ascending" net (ascending in terms of the "above" phrasing we just introduced), by taking the inclusion map $i$ from $(C, \leqslant)$ into $W$, which preserves the order; that is, $x \leqslant y$ in the domain $(C, \leqslant)$ means that $y$ is "after" $x$, whereas $x \leqslant y$ in $(W, \leqslant)$ means that $y$ is "above" $x$, so that $C$ goes up and up. Therefore, $C$ (as an ascending net) converges in $\mathfrak{I}(W)$ to $e \in W$ if, for every $U \in \mathfrak{I}(W)$ containing $e$, there is $x \in C$ such that $y=i(y) \in U$ for every $y \in C$ "after" $x$ (meaning, $y \geqslant x$ ), that is, $x \in C$ such that $C^{\geqslant x} \subseteq U$.

$C$ can also be regarded as a "descending" net, by taking the inclusion map $i$ from $(C, \geqslant)$ into $W$, which reverses the order; that is, $x \geqslant y$ in $(C, \geqslant)$ means that $y$ is "after" $x$, whereas $y \leqslant x$ in $(W, \leqslant)$ means that $y$ is "below" $x$, so that $C$ goes down and down. Therefore, $C$ (as a descending net) converges in $\mathfrak{I}(W)$ to $e \in W$ if, for every $U \in \mathfrak{I}(W)$ containing $e$, there is $x \in C$ such that $y=i(y) \in U$ for every $y \in C$ "after" $x$ (this time meaning $y \leqslant x$ ), that is, $x \in C$ such that $C^{\leqslant x} \subseteq U$.

15 In general, antisymmetry is not assumed on the order $\leqslant$ on $D$. See, e.g., Willard (1970, pp. 73-77). 
Lemma 48 Fix a history $h$, and let $X$ be either $h$ or $W$. Then, given any chain $C \subseteq h$, both of the following hold.

1. C regarded as a descending net converges in $\mathfrak{I}(X)$ to $\inf (C)$ if it exists.

2. $C$ regarded as an ascending net converges in $\mathfrak{I}(X)$ to $\sup _{h}(C)$ if it exists.

Proof Ad 1. Suppose $\inf (C)$ exists. We show that $C$ (as a descending net) converges in $\mathfrak{I}(X)$ to $\inf (C)$, which means that, for every $U \in \mathfrak{I}(X)$ containing $\inf (C)$, there is $x \in C$ such that $C^{\leqslant x} \subseteq U$.

Fix any $U \in \mathfrak{I}(X)$ containing $\inf (C)$. If $\inf (C)$ is minimal in $X$ then $U=X$ and hence $C^{\leqslant x} \subseteq U$ for any $x \in C(C \neq \varnothing \operatorname{since} \inf (C)$ exists $)$; so suppose $\inf (C)$ is not minimal in $X$. Since $\inf (C) \leqslant C$, there is $t \in M C(h)$ containing both $C$ and $\inf (C)$; therefore $\inf (C) \in U \in \mathfrak{I}(X)$ means that there are $z, y \in t$ such that $z<\inf (C)<y$ and $d^{z y} \subseteq U$ (such $z$ exists since $\inf (C)$ is not minimal in $X$ ). Then there is $x \in C$ such that $x \leqslant y$ (for otherwise $y \leqslant C$ and hence $y \leqslant \inf (C)$ ). This and $z<\inf (C) \leqslant$ $C$ imply $C^{\leqslant x} \subseteq d^{z y} \subseteq U$. Thus $C$ converges to $\inf (C)$ in $\mathfrak{I}(X)$.

A proof that is symmetrical (except dropping the case of minimal $\inf (C)$ ) would verify (2).

Note that a topology is Hausdorff if and only if every net in it converges to at most one point. Therefore Lemma 48.2 with $X=W$ gives another proof that $\mathfrak{I}(W)$ fails to be Hausdorff when a modal fork $t_{1}, t_{2}\left(t_{1} \in M C\left(h_{1}\right), t_{2} \in M C\left(h_{2}\right)\right)$ exhibits passive indeterminism, because then the trunk $C=t_{1} \cap t_{2}$ converges to two distinct points, namely, $\sup _{h_{1}}(C) \neq \sup _{h_{2}}(C)$.

Theorem 49 Suppose a history h has no minimal elements. Then, given any chain $C \subseteq h$ and $e \in h$, both of the following hold.

1. $C$ regarded as a descending net converges in $\mathfrak{I}(h)$ to $e$ iff $e=\inf (C)$.

2. $C$ regarded as an ascending net converges in $\mathfrak{I}(h)$ to $e$ iff $e=\sup _{h}(C)$.

Proof Ad 1. The "if" direction is just Lemma 48.1. For the "only if" direction, suppose $C$ converges in $\mathfrak{I}(h)$ to $e$.

Suppose for contradiction that $e \not C$; then $e \not x$ for some $x \in C$. By Lemma 36, $e \in \emptyset(x) \cap h \in \mathfrak{I}(h)$ (since $e$ is not minimal), whereas $C^{\leqslant x} \leqslant x$ means ( $(x) \cap h) \cap C^{\leqslant x}=\varnothing$, contradicting $C$ converging to $e$. Thus $e \leqslant C$.

The convergence of $C$ entails $C \neq \varnothing$; so the lowerbounded $C$ has $\inf (C)$. If $\inf (C) \notin e$, then $e \in M(\inf (C)) \cap h \in \mathfrak{I}(h)$ by Lemma 31, whereas $\inf (C) \leqslant C$ means $(\mathrm{M}(\inf (C)) \cap h) \cap C=\varnothing$, contradicting $C$ converging to $e$; so $\inf (C) \leqslant e$. Hence $\inf (C)=e$, because $e \leqslant C$ implies $e \leqslant \inf (C)$.

A symmetrical proof would verify (2).

Theorem 50 Suppose $W$ satisfies $C 1-C 4$. Then, given any chain $C$ and $e \in W$, both of the following hold.

1. C regarded as a descending net converges in $\mathfrak{I}(W)$ to e iff $e=\inf (C)$.

2. $C$ regarded as an ascending net converges in $\mathfrak{I}(W)$ to $e$ iff $e=\sup _{h}(C)$ for some history $h$. 
Proof Ad 1. The "if" direction is just Lemma 48.1. For the "only if" direction, suppose $C$ as a descending net converges in $\mathfrak{I}(W)$ to $e$, and pick any histories $h_{1}$ and $h_{2}$ containing $e$ and $C$, respectively. Applying $C 3$, pick $t_{1} \in M C\left(h_{1}\right)$ such that $e \in t_{1}$ and $t_{1}<e \neq \varnothing$ but $t_{1}^{<e} \cap$ blc $_{h_{1}}(e)=\varnothing$.

We first claim that, for any $x \in C$, if $e \not x$ then $\sup _{h_{2}}\left(t_{1}<e\right) \leqslant x$. Assuming $e \not x$ for $x \in C$, suppose for contradiction that $t_{1}<e \not x$; that is, $e_{0} \nless x$ for some $e_{0} \in t_{1}<e$. Then $t_{1}<e \cap b l c_{h_{1}}(e)=\varnothing$ implies $e \in \nabla\left(e_{0}\right)$ by $C 1$, whereas $\nabla\left(e_{0}\right) \in \mathfrak{I}(W)$ by $C 2$, $C 4$ and Corollary 29. On the other hand, $C^{\leqslant x} \cap \nabla\left(e_{0}\right)=\varnothing$ because $e_{0} \nless x$ implies $e_{0} \nless y$ for every $y \leqslant x$. These contradict since $C$ converges to $e$. Therefore $t_{1}<e \leqslant x$. This implies $\sup _{h_{2}}\left(t_{1}<e\right) \leqslant x$ since $t_{1}<e \neq \varnothing$ and $x \in C \subseteq h_{2}$.

Now suppose for contradiction that $e \not C$; that is, $e \not x$ for some $x \in C$. Then, indeed, $e \not z y$ for every $y \in C^{\leqslant x}$; hence the claim we showed above implies $\sup _{h_{2}}\left(t_{1}<e\right) \leqslant C^{\leqslant x}$. Therefore $M\left(\sup _{h_{2}}\left(t_{1}^{<e}\right)\right) \cap C^{\leqslant x}=\varnothing$. On the other hand, $e \in M$ $\left(\sup _{h_{2}}\left(t_{1}<e\right)\right) \in \mathfrak{I}(W)$ (by Corollary 32), because $e=\sup _{h_{1}}\left(t_{1}<e\right) \neq \sup _{h_{2}}\left(t_{1}<e\right) \leqslant x$ implies $\sup _{h_{2}}\left(t_{1}<e\right) \notin e$. These contradict since $C$ converges to $e$. Thus $e \leqslant C$.

The convergence of $C$ entails $C \neq \varnothing$; so the lowerbounded $C$ hasinf $(C)$. If $\inf (C) /$ $\leqslant e$ then $e \in M(\inf (C)) \in \mathfrak{I}(W)$ (by Corollary 32), whereas $\inf (C) \leqslant C$ means $M$ $(\inf (C)) \cap C=\varnothing$, contradicting $C$ converging to $e$; so $\inf (C) \leqslant e$. Hence $\inf (C)=e$, because $e \leqslant C$ implies $e \leqslant \inf (C)$.

$A d 2$. The "if" direction is just Lemma 48.2. For the "only if" direction, suppose $C$ as an ascending net converges in $\mathfrak{I}(W)$ to $e$.

Suppose for contradiction that $C \not e$; that is, $x \notin e$ for some $x \in C$. This means $e \in$ $M(x) \in \mathfrak{I}(W)$ (by Corollary 32), whereas $x \leqslant C^{\geqslant x}$ means $M(x) \cap C^{\geqslant x}=\varnothing$, contradicting $C$ converging to $e$. Thus $C \leqslant e$.

The convergence of $C$ entails $C \neq \varnothing$; so, for $h \in H_{(e)}, C \leqslant e \in h$ implies $C \subseteq h$ and that $\sup _{h}(C)$ exists. Suppose for contradiction that $e \notin \sup _{h}(C)$. Then, since $e, \sup _{h}(C) \in h$, Lemma 34 implies by $C 1, C 3$ that $e \in \nabla(x)$ and $x \notin \sup _{h}(C)$ for some $x \in h$. By $C 2, C 4$ and Corollary $29, \nabla(x) \in \mathfrak{I}(W)$, whereas $x \not \sup _{h}(C)$ implies $x \leqslant y$ for no $y \in C$ and hence $\nabla(x) \cap C=\varnothing$, contradicting $C$ converging to $e$. Thus $e \leqslant \sup _{h}(C)$. Therefore $e=\sup _{h}(C)$ because $C \leqslant e \in h$ and $\sup _{h}(C) \leqslant e$.

\subsection{Branching for the Laplaceans}

This subsection dwells on a consequence of Theorem 7, namely that the Bartha topology $\mathfrak{I}(W)$ on Our World can be regarded as naturally constructed from the Bartha topologies $\mathfrak{I}(h)$ on the histories $h$; so, to the extent that $\mathfrak{I}(h)$ are natural, the naturalness propagates to $\mathfrak{I}(W)$. This naturalness is based on "universal mapping properties," and applies not just to the theory of BST but also to the Laplacean diverging spacetimes, in the following manner: Given an ensemble of spacetimes that comes with a criterion of "identifying" points of different spacetimes, we can think of a branching structure constructed by naturally gluing those spacetimes together at identified points. This branching structure is to the diverging spacetimes what a BST structure $W$ is to its histories $h$ (we will show in which sense). Then Theorem 7 points to how, given topologies on branching histories or diverging spacetimes, to naturally 
construct from them a topology on the branching structure, whether it is a BST branching or an ensemble branching.

We first lay out a natural construction in which to glue spacetimes (as sets) together into one branching structure (as a set). Suppose, along the line of "ensemble branching" (see Sect. 2), that we are given an ensemble Hist of spacetimes $h$ and a criterion for "identifying" points of different spacetimes (for instance, sharing the same genuine physical magnitudes). Formally, take the disjoint union $\sum_{h} h$ of histories and write $x R y$ to mean that $x, y \in \sum_{h} h$ are "identified." The disjoint union $\sum_{h} h$ can simply be the union $\bigcup_{h} h$ if all histories are mutually disjoint (as in Lewis's "divergence"); otherwise, we label points of histories and let $\sum_{h} h=\{(e, h) \mid e \in h \in H i s t\}$. Finally, let $W$ be the quotient of $\sum_{h} h$ by the relation $R .{ }^{16}$ Let us call the set $W$ constructed in this way from a given ensemble Hist with a given relation $R$ (on $\sum_{h} h$ ) the branching structure for Hist and $R$; in short, $W$ is an ensemble Hist of spacetimes glued together at points identified by a relation $R$.

This "gluing" construction gives us the natural structure of branching, in the following sense. Let us express the construction in terms of the following two sorts of maps: One is the family of inclusion maps $m_{h^{\prime}}: h^{\prime} \rightarrow \sum_{h} h$, each of which maps $e \in h^{\prime}$ to $\left(e, h^{\prime}\right)$ (or to $e$ itself if $h$ are all disjoint). The other is the quotient map $q$ : $\sum_{h} h \rightarrow W$, which maps $x \in \sum_{h} h$ to the equivalence class $[x]$ of points identified with $x$. Then the construction of the branching structure yields the following "universal mapping property": ${ }^{17}$

Fact 51 Given a family Hist and a relation $R$ on $\sum_{h} h$ (which may or may not be an equivalence relation), the branching structure $W$ for Hist and $R$, with maps $m_{h}: h$ $\rightarrow \sum_{h} h$ and $q: \sum_{h} h \rightarrow W$, is the unique (up to isomorphism) set that satisfies the following.

1. W identifies all that has to be identified; that is, if $R$ identifies $e_{1} \in h_{1}$ and $e_{2} \in h_{2}$-in the sense that $m_{h_{1}}\left(e_{1}\right) R m_{h_{2}}\left(e_{2}\right)$-then $q \circ m_{h_{1}}\left(e_{1}\right)=q \circ m_{h_{2}}\left(e_{2}\right)$ $\in W$.

2. For any set $X$ and a family of maps $f_{h}: h \rightarrow X(h \in$ Hist, $)$ if the family $f_{h}$ respects $R$-in the sense that $f_{h_{1}}\left(e_{1}\right)=f_{h_{2}}\left(e_{2}\right)$ whenever $m_{h_{1}}\left(e_{1}\right) R m_{h_{2}}\left(e_{2}\right)$-then there is a unique map $f: W \rightarrow X$ such that $f \circ q \circ m_{h}=f_{h}$ for all $h \in$ Hist.

This fact can also be put as follows:

Corollary 52 Let $W$ be the branching structure for a family Hist of sets and a relation $R$ on $\sum_{h} h$. Then, for any set $X$, the families of maps $f_{h}: h \rightarrow X(h \in$ Hist $)$ that respect $R$ correspond one-to-one to the maps $f: W \rightarrow X$.

Proof For the correspondence "to the right," associate with a given $R$-respecting family $f_{h}: h \rightarrow X$ the map $f: W \rightarrow X$ as in Fact 51.2. "To the left," associate with given $f: W \rightarrow X$ the family $f \circ q \circ m_{h}: h \rightarrow X$, which respects $R$ by Fact 51.1. And the correspondence is bijective by Fact 51.2.

\footnotetext{
${ }^{16}$ When $R$ is not itself an equivalence relation, by "the quotient by $R$ " we mean the quotient by the equivalence relation that $R$ generates.

17 See Brown (2006, p. 100), for a proof (of essentially the same fact). The results that follow are essentially ones laid out in Brown (2006, pp. 100f.).
} 
These results mean the following. We may wish to assign some value (a vector, for instance) to each point of every spacetime $h$; that is to take a family of maps $f_{h}$ : $h \rightarrow X$ (say, to a vector space $X$ ). Then, as long as this assignment is coherent with the relevant criterion of identifying points of different spacetimes (for instance, assigning the same vector to points sharing the same physical magnitudes), $f: W \rightarrow$ $X$ as in Fact 51.2 gives an equivalent description of the assignment-carrying all and only the information carried by the family $f_{h}$-in the form of a single map. And $W$ is the only set that provides the domain for such a corresponding map $f$.

It is worth noting that, given a BST model $(W, \leqslant)$, the set $W$ is (isomorphic to) the branching structure, in the sense we defined above, for its set of histories Hist and the obvious relation $R$ of identification (that is, $\left(e_{1}, h_{1}\right) R\left(e_{2}, h_{2}\right)$ iff $e_{1}=e_{2}$ ); then each $q \circ m_{h}$ is just the inclusion map $i_{h}: h \rightarrow W$, which sends $e \in h$ to $m_{h}(e)=(e, h)$ to $q(e, h)=e .^{18}$ In short, $W$ is "reconstructed" by first labeling events and then dropping the labels. Hence, $W$ with $m_{h}$ and $q$ satisfies Fact 51 and Corollary 52 for its histories Hist and the obvious $R$.

Let us now turn to Theorem 7. We can read it as follows: The Bartha topology $\mathfrak{I}(W)$ on Our World, which is concretely defined in terms of diamonds $d^{x y}$, can also be constructed from the family of Bartha topologies $\mathfrak{I}(h)$ on histories $h \in$ Hist by setting $\mathfrak{I}(W)=\{A \subseteq W \mid A \cap h \in \mathfrak{I}(h)$ for all $h \in H i s t\}$, or, in other words, as the finest topology on $W$ that has each inclusion map $i_{h}: h \rightarrow W$ continuous (note that $A$ $\cap h=i_{h}^{-1}[A]$ for $A \subseteq W ; \mathfrak{I}(W)$ is the finest because, if another topology $\mathfrak{I}_{0}$ on $W$ has all $i_{h}$ continuous, then every $A \in \mathfrak{I}_{0}$ has $A \cap h=i_{h}{ }^{-1}[A] \in \mathfrak{I}(h)$ for all $h \in$ Hist and hence $A \in \mathfrak{I}(W)$, that is, $\mathfrak{I}_{0} \subseteq \mathfrak{I}(W)$ ). This construction is a common one in topology, a particular case of the construction of gluing several spaces (not just as sets but as sets plus topologies) together into one. ${ }^{19}$

This construction can indeed be generalized and applied to any branching structure $W$-not only of BST histories but also of divergent spacetimes-by replacing the inclusion maps $i_{h}$ with maps $q \circ m_{h}$ in general, as follows. Let $\mathfrak{I}\left(\sum_{h} h\right)$ be the "disjoint union topology," that is, the finest topology on $\sum_{h} h$ that makes all $m_{h}$ continuous-more concretely, $\mathfrak{I}\left(\sum_{h} h\right)=\left\{B \subseteq \sum_{h} h \mid m_{h^{\prime}}{ }^{-1}[B] \in\right.$ $\mathfrak{I}\left(h^{\prime}\right)$ for all $h^{\prime} \in$ Hist $\}$. Then let $\mathfrak{I}(W)$ be the "quotient topology," that is, the finest topology on $W$ that makes $q$ continuous- $\mathfrak{I}(W)=\left\{A \subseteq W \mid q^{-1}[A] \in \mathfrak{I}\left(\sum_{h} h\right)\right\}=$ $\left\{A \subseteq W \mid\left(q \circ m_{h}\right)^{-1}[A] \in \mathfrak{I}(h)\right.$ for all $\left.h \in H i s t\right\}$. Thus any branching structure $W$, whether it is a BST one or a diverging one, accommodates the gluing construction of $\mathfrak{I}(W)$ from $\mathfrak{I}(h)$. Let us call such $\mathfrak{I}(W)$ the branching topology for Hist, $R$ and the family of topologies $\mathfrak{I}(h)$.

The branching topology $\mathfrak{I}(W)$ constructed on a branching structure $W$ in the manner above is the natural topology on $W$ for the following reason. Recall that, given any ensemble Hist of histories or spacetimes (whether it comes from a BST

\footnotetext{
18 Strictly speaking, $W$ is not itself the branching structure in the defined sense: $W$ is a set of events, whereas we take as the branching structure a set of equivalence classes of event-history pairs; so $q(e, h)$ is not $e$, but rather $\left\{\left(e, h^{\prime}\right) \mid e \in h^{\prime} \in H i s t\right\}=\{e\} \times H_{(e)}$. Yet $W$ is isomorphic to the branching structure: $e$ and $\{e\} \times H_{(e)}$ are in an obvious one-to-one correspondence.

19 For a variety of such constructions, see, e.g., Willard (1970, pp. 59-69), and Brown (2006, pp. 97-105).
} 
structure or from divergence) and any relation $R$ on $\sum_{h} h$ (that serves as a criterion for identifying points of different spacetimes), the naturalness of the branching structure $W$ for Hist and $R$ is captured by the correspondence stated in Fact 51 and Corollary 52. This correspondence, on the level of sets and maps, extends to the topological level:

Fact 53 Let the set $W$ be the branching structure (as a set) for a family Hist of topological spaces and a relation $R$ on $\sum_{h} h ;$ write $\mathfrak{I}(h)$ for the topology on $h \in$ Hist. Given any topological space $X$ and a family of maps $f_{h}: h \rightarrow X(h \in H i s t)$ that respects $R$, consider the map $f: W \rightarrow X$ given by Fact 51.2. Then $\mathfrak{I}(W)$ is the unique topology on W that satisfies: $(*)$ fis continuous iff all $f_{h}$ are continuous (with $\mathfrak{I}(h)$ ).

Proof The "only if" part of $(*)$ holds for $\mathfrak{I}(W)$ because $f_{h}=f \circ q \circ m_{h}$ while the composition of continuous maps is continuous ( $q$ and $m_{h}$ are continuous).

For the "if" part of $(*)$ for $\mathfrak{I}(W)$, suppose $f_{h}$ are all continuous and fix any open subset $U$ of $X$. Then, for each $h \in$ Hist, the continuity of $f_{h}$ implies $f_{h}{ }^{-1}[U] \in \mathfrak{I}(h)$, whereas $f_{h}^{-1}[U]=\left(f \circ q \circ m_{h}\right)^{-1}[U]=\left(q \circ m_{h}\right)^{-1}\left[f^{-1}[U]\right]$. Thus $\left(q \circ m_{h}\right)^{-1}\left[f^{-1}[U]\right]$ $\in \mathfrak{I}(h)$ for all $h \in$ Hist, which means $f^{-1}[U] \in \mathfrak{I}(W)$ by the construction of $\mathfrak{I}(W)$.

For the uniqueness part, fix any topology $\mathfrak{I}_{0}$ on $W$ that satisfies $(*)$. Let us take $q \circ m_{h}: h \rightarrow W$ as $f_{h}: h \rightarrow X$; then $f$ is the identity map $i: W \rightarrow W$. Since $f=i$ is continuous from $\mathfrak{I}_{0}$ to $\mathfrak{I}_{0}$, the "only if" part of $(*)$ for $\mathfrak{I}_{0}$ implies that all $f_{h}=$ $q \circ m_{h}$ are continuous from $\mathfrak{I}(h)$ to $\mathfrak{I}_{0}$. Therefore the "if" part of $(*)$ for $\mathfrak{I}(W)$ implies that $f=i$ is continuous from $\mathfrak{I}(W)$ to $\mathfrak{I}_{0}$, which means $\mathfrak{I}_{0} \subseteq \mathfrak{I}(W)$ because $i^{-1}[U]=U$. On the other hand, the "if" part of $(*)$ for $\mathfrak{I}_{0}$ implies that $f=i$ is continuous from $\mathfrak{I}_{0}$ to $\mathfrak{I}(W)$, that is, $\mathfrak{I}(W) \subseteq \mathfrak{I}_{0}$. Thus $\mathfrak{I}_{0}=\mathfrak{I}(W)$.

While we may express assignment of values (or vectors, etc.) to points of spacetimes $h$ by a family of maps $f_{h}: h \rightarrow X$, it may then be desirable to distinguish continuous assignments of values from non-continuous ones. This distinction is made by the topologies $\mathfrak{I}(h)$ (as well as by the topology on $X$ ). Recall that, when such an assignment is coherent with the criterion of identifying points of different spacetimes, it is equivalently described by a single map $f: W \rightarrow X$. Then Fact 53 means that $\mathfrak{I}(W)$ is the only topology that agrees with the family $\mathfrak{I}(h)$ regarding whether the assignment is continuous or not. ${ }^{20}$

In sum, these facts establish that, given an ensemble Hist of histories $h$ and their topologies $\mathfrak{I}(h)$, the branching structure $W$ and the branching topology $\mathfrak{I}(W)$ obtained with the gluing constructions are the natural structure of branching for Hist, whether the family Hist comes from a BST model or from divergence. These

\footnotetext{
${ }^{20}$ It may be worth noting that the gluing construction can also be extended to orderings on the histories $h$. Suppose we are given a set Hist of preordered sets $\left(h, \lesssim_{h}\right)$ and a relation $R$ on $\sum_{h} h$. (A preorder is a reflexive and transitive relation, that is, a partial order without antisymmetry assumed.) On the branching structure $W$ for Hist and $R$, we can define the "branching order for the family $\lesssim_{h}$ " as the smallest preorder on $W$ with which all $q \circ m_{h}$ preserve order. Then the preorder version of Fact 53 is available. The BST ordering on any BST model $(W, \leqslant)$ is in fact the branching order, in this sense, for the family of its restrictions to histories $h$. We should however note that, in non-BST cases, this construction works for preorders but not necessarily for partial orders: There may be no partial order on the branching structure with which all $q \circ m_{h}$ preserve order (whether $\lesssim_{h}$ are partial orders or just preorders).
} 
two constructs are characterized by the two universal mapping properties expressed by Facts 51 through 53-one of them assures a correspondence between maps $f$ and families of maps $f_{h}$, while the other assures their agreement on continuity. The constructions are so natural that any theory of branching, whether it is BST or of ensemble-branching type, would have to admit them.

This does not mean that any theory of branching of spacetimes, if guided by the requirement of naturalness, must satisfy the BST axioms and adopt Bartha topologies. We merely claimed that the gluing constructions are natural in the sense that, given any histories $h$ and topologies $\mathfrak{I}(h)$ on them, their branching structure $W$ and branching topology $\mathfrak{I}(W)$ should be in a certain relation with them. This has no consequences for other features of the theory of BST-for instance, the prior choice principle. An ensemble branching with a significantly different criterion $R$ of identifying points of spacetimes, or with significantly different topologies, although it has to construct $W$ and $\mathfrak{I}(W)$ by gluing, may end up with significantly different features of branching and topology on $W$. In a slogan, branching is everywhere, though it might be different from the BST branching.

\section{Conclusions}

We began this study by inquiring whether Belnap's (1992) theory of branching spacetimes satisfies the critical Hausdorff property. To ask a sensible question required both a rigorous account of $\mathrm{BST}$ models $\langle W, \leqslant\rangle$ and a reasonable topology for Our World, $W$, and for each of its histories, $h$. The definition of BST models came from Belnap (1992), and to satisfy the second requirement, we adopted the "Bartha topology," aka "the diamond topology," as the best choice, both for defining the class $\mathfrak{I}(W)$ of open sets of $W$ and the class $\mathfrak{I}(h)$ of open sets of each history, $h$. Our Theorem 7 shows then a relation between open sets of $W$ and open sets of $h$.

Next we defined light-cones in BST models, and to guarantee that they have properties similar to those of light-cones of space-times of physics, we assumed four natural postulates $C 1-C 4$ on branching histories. We proved that light-cones are history-independent, which provides evidence that our definitions properly generalize the concept of light-cones from a single space-time to a modal context of many branching histories. We showed that provided we rule out certain pathological models by the addition of conditions $C 1-C 4$ to the postulates of BST, the set of "borderless diamonds" serves as a base for the topology $\mathfrak{I}(h)$ (Theorem 28).

With these materials in hand, we turned to our first question: Do BST histories have the Hausdorff property? The most important result of this investigation is that the answer turns positive when we ask if the four conditions $C 1-C 4$ suffice for the Hausdorff property for individual BST histories (Theorem 35). Since in our manybranching-histories representation of indeterminism, each BST history was intended to play the role of a spacetime, and since most spacetime theorists believe that a topological understanding of spacetime cries out for the Hausdorff property, Theorem 35, with its intricate proof, was welcome indeed. 
Turning to our central question of what regions of chanciness look like, we limited our attention to the study of the Hausdorff property in the world of branching histories. Our main results, Theorems 42 and 47, relate the satisfaction of the Hausdorff property in a BST model to the existence of passive indeterminism in the model. Theorem 42 tells us that the failure of the Hausdorff property is a necessary condition of passive indeterminism. Theorem 47 tidies up our field of concepts by showing that given the conditions $C 1-C 4$, the implication can be strengthened to an equivalence: Passive indeterminism and the failure of the Hausdorff property stand or fall together. One should infer that whereas the Hausdorff property rightly seems essential for any reasonable theory of spacetime, on the other hand, it seems totally out of place for a world consisting of a family of branching spacetimes.

The equivalence of a failure of the Hausdorff property and passive indeterminism prompted our final question: Just how ubiquitous is passive indeterminism? The answer is given by our Lemma 43: Excluding some pathology, an indeterministic BST model (i.e., a model with choice points) exhibits passive indeterminism.

We read these results as optimistic, especially for a future project of merging branching space-times with general relativity. Given physically realistic conditions $C 1-C 4$, each history (or space-time) has the Hausdorff property, which is physically important. Whether this property is satisfied by a whole model of branching histories is neutral to current physics. One may thus attempt to associate a differential structure to a BST model in such a way that each history be a timeoriented manifold satisfying the Hausdorff property. ${ }^{21}$

Acknowledgments We are grateful to Thomas Müller and two anonymous referees for comments on this paper. TP's research has been supported by the research grant 668/N-RNP-ESF/2010/0 of the (Polish) Ministry of Science and Higher Education. KK's research has been funded by the VIDI grant 639.072.904 of the Netherlands Organization for Scientific Research (NWO).

Open Access This article is distributed under the terms of the Creative Commons Attribution License which permits any use, distribution, and reproduction in any medium, provided the original author(s) and the source are credited.

\section{References}

Belnap, N. (1992). Branching space-time. Synthese, 92, 385-434. (See also Belnap (2003a).)

Belnap, N. (2003a). Branching space-time, postprint, January, 2003. http://philsci-archive.pitt.edu/ archive/00001003/.

Belnap, N. (2003b). No-common-cause EPR-like funny business in branching space-times. Philosophical Studies, 114, 199-221.

Belnap, N. (2005). A theory of causation: Causae causantes (originating causes) as inus conditions in branching space-times. British Journal for the Philosophy of Science, 56, 221-253. (A preprint of this essay may be obtained from http://philsci-archive.pitt.edu/archive/00000891/.)

Brown, R. (2006). Topology and groupoids. http://www.groupoids.org, Deganwy.

\footnotetext{
${ }^{21}$ In this context we mention a somewhat different project of Müller (2011) that modifies BST axioms to assure that a BST model (as a whole) is a generalized manifold (i.e., Euclidean but not necessarily Hausdorff).
} 
Earman, J. (1986). A primer on determinism. Dordrecht: D. Reidel Publishing Company.

Earman, J. (2008). Pruning some branches from branching spacetimes. In D. Dieks (Ed.), The ontology of spacetime II, chapter 10 (pp. 187-206). Amsterdam: Elsevier.

Laplace, P. S. (1820/1902). A philosophical essay on probabilities. London: Wiley.

Lewis, D. (1983). New work for a theory of universals. Australasian Journal of Philosophy, 61, 343-377.

Mackie, J. L. (1974). The cement of the universe. Oxford: Oxford University Press.

McCabe, G. (2005). The topology of branching universes. Foundations of Physics Letters, 18(7), 665-676.

Montague, R. (1962). Deterministic theories. In N. F. Washburne (Ed.), Decisions, values, and groups 2, (pp. 325-370). Oxford: Oxford University Press. (Reprinted in his Formal philosophy: selected papers of Richard Montague. Yale University Press, New Haven 1974. Edited and with an introduction by R. H. Thomason).

Müller, T. (2002). Branching space-time, modal logic and the counterfactual conditional. In T. Placek \& J. Butterfield (Eds.), Nonlocality and Modality, NATO Science Series (pp. 273-291). Dordrecht: Kluwer.

Müller, T. (2009). Eliminating modality from the determinism debate? Models vs. equations of physical theories. In A. Hieke \& H. Leitgeb (Eds.), Reduction-abstraction-analysis. Proceedings of the 31st international Ludwig Wittgenstein-symposium in Kirchberg, 2008 (pp. 47-62). Frankfurt: Ontos Verlag.

Müller, T. (2011). Branching space-times, general relativity, the Hausdorff property, and modal consistency, technical report, http://philsci-archive.pitt.edu/8577/.

Øhrstrøm, P., \& Hasle, P. F. V. (1995). Temporal logic: From ancient ideas to artificial intelligence. Dordrecht: Kluwer.

Penrose, R. (1979). Singularities and time-asymmetry. In: S. W. Hawking \& W. Israel (Eds.), General relativity: An Einstein centenary survey (pp. 581-683). Cambridge: Cambridge University Press.

Placek, T., \& Belnap, N. (2012). Indeterminism is a modal notion: Branching spacetimes and Earman's pruning. Synthese, 187, 441-469. doi:10.1007/s11229-010-9846-8.

Prior, A. N. (1967). Past, present, and future. Oxford: Oxford University Press.

Thomason, R. H. (1970). Indeterminist time and truth-value gaps. Theoria, 36, 264-281.

Wald, R. M. (1984). General relativity. Chicago: University of Chicago Press.

Weiner, M., \& Belnap, N. (2006). How causal probabilities might fit into our objectively indeterministic world. Synthese, 149(1), 1-36.

Willard, S. (1970). General topology. Reading: Addison-Wesley Publishing Company. (Reprinted (2004). Mineola: Dover Publications.)

Wroński, L., \& Placek, T. (2009). On Minkowskian branching structures. Studies in History and Philosophy of Modern Physics, 40, 251-258. 